This item was submitted to Loughborough's Research Repository by the author.

Items in Figshare are protected by copyright, with all rights reserved, unless otherwise indicated.

\title{
Focus variation microscope: linear theory and surface tilt sensitivity
}

PLEASE CITE THE PUBLISHED VERSION

http://dx.doi.org/10.1364/AO.55.003555

PUBLISHER

(C) Optical Society of America

\section{VERSION}

AM (Accepted Manuscript)

\section{PUBLISHER STATEMENT}

This work is made available according to the conditions of the Creative Commons Attribution-NonCommercialNoDerivatives 4.0 International (CC BY-NC-ND 4.0) licence. Full details of this licence are available at: https://creativecommons.org/licenses/by-nc-nd/4.0/

\section{LICENCE}

CC BY-NC-ND 4.0

\section{REPOSITORY RECORD}

Nikolaev, Nikolay, Jon Petzing, and Jeremy Coupland. 2016. "Focus Variation Microscope: Linear Theory and Surface Tilt Sensitivity". Loughborough University. https://hdl.handle.net/2134/21628. 


\title{
The Focus Variation Microscope: Linear Theory and Surface Tilt Sensitivity
}

\author{
Nikolay Nikolaev, Jon Petzing and Jeremy Coupland * \\ Mechanical and Manufacturing Engineering, Loughborough University, LE11 3TU, UK \\ *Corresponding author: j.m.coupland@lboro.ac.uk
}

Received XX Month XXXX; revised XX Month, XXXX; accepted XX Month XXXX; posted XX Month XXXX (Doc. ID XXXXX); published XX Month XXXX

\begin{abstract}
In a recent publication [3rd International Conference on Surface Metrology, Annecy, France, 2012, p. 1] it was shown that surface roughness measurements made using a focus variation microscope (FVM) are influenced by surface tilt. The effect appears to be most significant when the surface has microscale roughness $(\mathrm{Ra} \approx 50 \mathrm{~nm})$ that is sufficient to provide a diffusely scattered signal that is comparable in magnitude to the specular component. This paper explores, from first principles, image formation using the focus variation method. With the assumption of incoherent scattering, it is shown that the process is linear and the 3D point spread characteristics and transfer characteristics of the instrument are well defined. It is argued that, for the case of micro-scale roughness and through the objective illumination, the assumption of incoherence cannot be justified and more rigorous analysis is required. Using a foil model of surface scattering the images that are recorded by a FVM have been calculated. It is shown that for the case of through the objective illumination at small tilt angles, the signal quality is degraded in a systematic manner. This is attributed to the mixing of specular and diffusely reflected components and leads to an asymmetry in the k-space representation of the output signals. It is shown that by using extra-aperture illumination or at tilt angles greater than the acceptance angle of aperture (such that the specular component is lost), the incoherent assumption can be justified once again. The work highlights the importance of using ring-light illumination and/or polarizing optics, which are often available as options on commercial instruments, as a means to mitigate or prevent these effects. (C) 2016 Optical Society of America.
\end{abstract}

OCIS codes: (180.0180) Microscopy; (110.4850) Optical transfer functions;

http://dx.doi.org/10.1364/AO.99.099999

\section{INTRODUCTION}

The focus variation microscope (FVM) is an increasingly popular means to measure the surface geometry of micro-components that compares favourably to the more established techniques of confocal microscopy (CM) and coherence scanning interferometry (CSI) [1]. The focus variation method exploits the limited depth of focus of a vertical scanning microscope and consequently is suited to the measurement of steep surfaces provided that they are optically rough. Like other optical methods, however, FVM measurements can be influenced by surface tilt and tilt-dependent surface roughness measurements have been recently reported [2]. In this paper we explain a basic 3D linear theory of the focus variation [3] and extend this concept to show the origin of tilt sensitivity in FVM.

\section{BASIC 3D LINEAR THEORY OF THE FOCUS VARIATION MICROSCOPE}

In previous publications we have analysed holography, tomography and 3D imaging techniques including CSI and CM using linear systems theory [4]. Linear systems theory allows us to characterise and compare optical systems in terms of their 3D point spread function (PSF), $H(\mathbf{r})$, that represents the response the instrument to a point object (or impulse) located at the origin. In this way the output, $O(\mathbf{r})$, of the instrument to more general objects, defined by the function, $\Delta(\mathbf{r})$, can be written as the superposition or convolution integral,

$$
O(\mathbf{r})=\int \Delta\left(\mathbf{r}^{\prime}\right) H\left(\mathbf{r}-\mathbf{r}^{\prime}\right) \mathrm{d}^{3} r^{\prime},
$$

where conventionally $\mathrm{d}^{3} r^{\prime}=\mathrm{d} r_{x}{ }^{\prime} d r_{y}{ }^{\prime} d r_{z}{ }^{\prime}$. In essence this is a linear filtering operation that can be represented in the frequency domain ( $k$-space) as the product,

$$
\tilde{O}(\mathbf{k})=\widetilde{\Delta}(\mathbf{k}) \widetilde{H}(\mathbf{k})
$$

where tilde denotes Fourier transformation. In this way $\widetilde{H}(\mathbf{k})$ denotes the transfer function (TF) and its extent defines the frequency response of the system while in the space domain that of the PSF defines the resolution. In general terms, the object function $\Delta(\mathbf{r})$ defines the object in $3 \mathrm{D}$ in terms of a physical parameter such as refractive index. For the case of 
surface scattering, however, we have found it useful to represent the object as a foil-like membrane located at the interface between media with optical properties characterised by the reflection coefficient [5]. We call this a "foil model" of the surface. In general terms, linear theories of optical measuring instruments all rest on the assumption that the effects of multiple scattering are negligible and further assumptions are inherent in the foil model of surface scattering [5].

Although the FVM is a surface measuring instrument, considerable insight into its performance can be gained without considering the details of surface scattering. A basic linear model of the focus variation method can be cast by considering the field scattered by the surface to be a set of independent, incoherent point sources of varying strength located on a foillike membrane. Accordingly it is intuitive to define the object function, $\Delta(\mathbf{r})$, such that,

$$
\Delta(\mathbf{r})=I_{s}(\mathbf{r}) \delta\left(r_{z}-s\left(r_{x}, r_{y}\right)\right),
$$

where $I_{S}(\mathbf{r})$ is a random function describing the source strength per unit area, $s\left(r_{x}, r_{y}\right)$ is the surface height, $\delta(x)$ is 1D Dirac delta function.

It is well known from 2D linear systems theory that the diffraction limited image of a point source is determined by the numerical aperture and is characterised by way of either its coherent or incoherent PSF [6]. The coherent PSF describes the phase and amplitude of the image of a point source and is particularly useful when coherent detection is used (i.e. digital holography). The incoherent PSF describes the intensity of this image and is the squared modulus of its coherent counterpart. It is straightforward to extend this concept into 3D in order to model the intensity recorded by a FVM as it scans through focus to form the so-called "image stack".

As noted previously [5] it is often more convenient to define the response of the system as the TF in the frequency domain (k-space). Accordingly the portion of the 3D field, $\tilde{G}_{O}(\boldsymbol{k})$, that can be collected (or measured) by any far-field instrument (including FVM, CSI etc.) of observational numerical aperture, No, can be written [5],

$$
\tilde{G}_{O}(\mathbf{k})=\frac{j}{4 \pi k_{0}} \delta\left(|\mathbf{k}|-k_{0}\right) \operatorname{step}\left(\frac{\mathbf{k} . \widehat{\mathbf{o}}}{k_{0}}-\sqrt{1-N_{O}^{2}}\right),
$$

where $k_{0}$ is the wavenumber, defined here as inverse wavelength such that $k_{0}=1 / \lambda, \delta(x)$ and step $(\mathrm{x})$ are the Dirac Delta and Heaviside Step functions respectively, while $\widehat{\mathbf{o}}$ is a unit vector in the direction of the optical axis of the instrument. It is noted that Eq. (4) defines a "cap-like" area of the surface of a sphere of radius $k_{0}=1 / \lambda$ and is a k-space representation of the 3D amplitude field collected by a far-field instrument due to a point source at the coordinate origin. Making use of the autocorrelation theorem [6], the corresponding intensity that describes the image recorded by a FVM can be expressed in the frequency domain by the auto-correlation function,

$$
\widetilde{H}_{B}(\mathbf{k})=\int \tilde{G}_{O}\left(\mathbf{k}^{\prime}\right) \tilde{G}_{O}^{*}\left(\mathbf{k}^{\prime}-\mathbf{k}\right) \mathrm{d}^{3} k^{\prime}
$$

It is interesting to note that this is the transfer function of a forward scatter, coherent confocal microscope $[4,7,8]$. If it is assumed that the optical axis of the instrument is in the $\mathrm{z}$ direction such that $\widehat{\mathbf{o}}=\mathbf{z}$, Eq. (5) can be integrated following the procedure presented in APPENDIX A, to give a closed form solution,

$$
\begin{gathered}
\widetilde{H}_{B}(\mathbf{k})=\frac{1}{8 \pi^{2}|\mathbf{k}|} \operatorname{step}\left(B^{2}\right) \tan ^{-1}\left(\frac{B}{\sqrt{k_{0}^{2}-|\mathbf{k}|^{2} / 4-B^{2}}}\right), \\
B=\sqrt{\left(k_{0}^{2}-|\mathbf{k}|^{2} / 4\right)-\left[\frac{|\mathbf{k}|}{2 \sqrt{k_{x}^{2}+k_{y}^{2}}}\left(\left|k_{z}\right|+2 k_{0} \sqrt{1-N_{O}^{2}}\right)\right]^{2}} .
\end{gathered}
$$

Under the assumption of incoherent scattering, this function, defines the TF that characterises the 3D image stack that is collected by a FVM as it scans through focus. The validity of this assumption will be discussed later, however, let us first consider the 3D form of the TF and the corresponding PSF with a view to identifying surface position.

A section through the TF defined by Eq. (6) is shown in figure 1a) for the case of a quasi-monochromatic imaging system with observational numerical aperture $\mathrm{N}_{0}=0.5$ operating at a nominal wavelength of $\lambda=0.5 \mu \mathrm{m}$. It is noted that the vertical extent of the TF $\left(\sim k_{0} N_{O}^{2}\right)$ is inversely proportional to the depth resolution while it's lateral extent $\left(\sim 4 k_{0} N_{O}\right)$ defines the lateral resolution of the instrument (by the Nyquist resolution criteria). Interestingly, this is exactly the Nyquist resolution offered by a coherent (interferometric) backscatter instrument, such as CSI or a coherent CM, of equal aperture [5].

By definition the inverse Fourier transform of Eq. (6) gives the PSF characteristic of incoherent imaging and a section through the corresponding PSF is shown in figure 1b). It can be seen that the PSF resembles a focussed beam and it is noted that, as such, it decays relatively slowly $\left(1 / z^{2}\right)$ in the axial direction. Using these characteristics 3D image of a plane surface can be calculated according to Eq. (2). An image section through such a surface is shown in figure 1c), where the source strength function, $I_{S}(\mathbf{r})$, is assumed to be white noise.

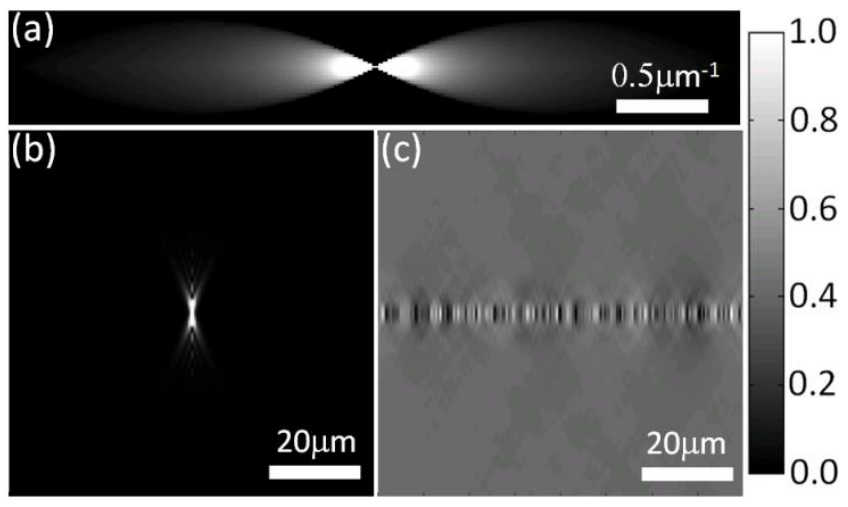

Fig. 1. Normalised images a) TF, b) PSF and c) surface image for instrument with numerical aperture $\mathrm{N}_{0}=0.5$ at nominal wavelength $\lambda=$ $0.5 \mu \mathrm{m}$ assuming incoherent scattering

Although several ways to deduce the position of a surface using incoherent imaging have been proposed in the literature $[2,9,10]$, we will not concern ourselves with the details of these here but merely note that the information that defines the position of the surface is found in the high frequency information contained in figure 1c). Accordingly the surface can be revealed by modifying the $\mathrm{TF}$ such that only these 
components are passed. In $3 \mathrm{D}$, a suitable filter, $F_{F V M}(\mathbf{k})$, is defined by the hollow cylinder,

$$
F_{F V M}(\boldsymbol{k})=\left\{\begin{array}{c}
1 \text { for } k_{\text {low }}<\sqrt{k_{x}^{2}+k_{y}^{2}}<k_{\text {high }}, \\
0 \text { otherwise }
\end{array}\right.
$$

where $k_{\text {low }}=0.6 k_{0} N_{0}$ and $k_{\text {high }}=1.5 k_{0} N_{0}$. The choice of $k_{\text {low }}$ and $k_{\text {high }}$ is somewhat arbitrary; in essence $F_{F V M}(\mathbf{k})$ selects the part of the transfer function that has the greatest axial extent while rejecting the uniform background intensity and low frequency components that contribute little to defining surface position. With this definition the TF of a filtered FVM, output becomes,

$$
\begin{aligned}
& \widetilde{H}_{F V M}(\mathbf{k})=F_{F V M}(\boldsymbol{k}) \widetilde{H}_{B}(\mathbf{k})= \\
= & \frac{F_{F V M}(\mathbf{k})}{8 \pi^{2}|\mathbf{k}|} \operatorname{step}\left(B^{2}\right) \tan ^{-1}\left(\frac{B}{\sqrt{k_{0}^{2}-|\mathbf{k}|^{2} / 4-B^{2}}}\right),
\end{aligned}
$$

where B is defined as before. The TF and PSF that characterise the output of a filtered FVM and the corresponding surface image are shown in figure 2 a)-c). In comparison with figures 1 a)-c) it is noted that although the lateral resolution is slightly decreased (because the lateral extent of the TF is reduced) the PSF is well defined in the axial direction and the surface is revealed as a distinct "string of beacons". Surface estimation can be considered to be the (non-linear) process of identifying and joining the centres of these "beacons".

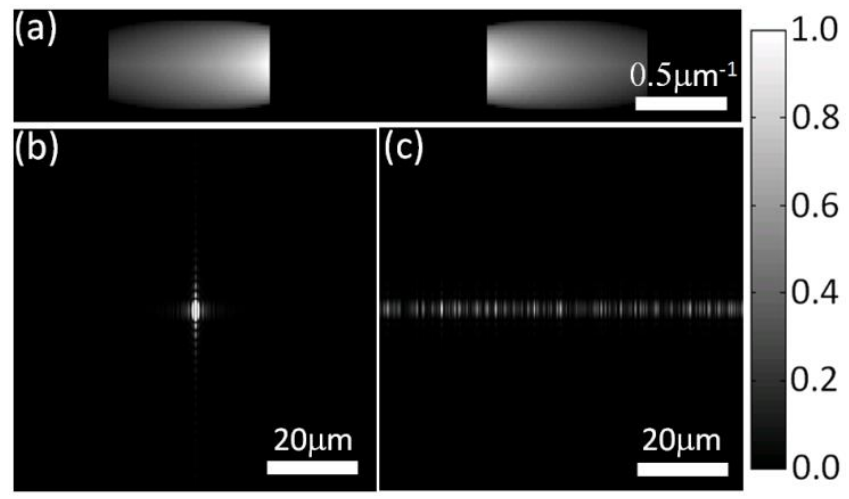

Fig. 2. Normalised images: a) Filtered TF, b) PSF and c) surface image for instrument with numerical aperture $\mathrm{N}_{0}=0.5$ at a nominal wavelength $\lambda=0.5 \mu \mathrm{m}$ assuming incoherent scattering

The underlying assumption that the scattered field is completely incoherent is only strictly true, however, if the illumination is spatially incoherent and/or the surface is sufficiently rough for multiple scattering to dominate. It is noted that illumination which propagates through the objective does not fulfil the former condition, while the latter is only satisfied when the $\mathrm{Ra} \gg \lambda$. Consequently many of the illumination conditions used in practice and many surfaces of interest do not satisfy this assumption. A more comprehensive analysis is therefore required.

\section{DETAILED ANALYSIS OF THE FOCUS VARIATION MICROSCOPE}

A more rigorous model of the FVM must take into account the characteristics of both the illumination and the surface scattering. The starting point for this analysis is a general expression for the field $E_{m}(\mathbf{r})$ collected (or measured) by an instrument with restricted numerical aperture, when the surface of interest is illuminated by a plane monochromatic wave of amplitude $E_{r}$. According to the analysis presented in reference [5] this is given by:

$$
\begin{gathered}
E_{m}(\mathbf{r})=E_{r} \iint e^{-2 \pi j\left(\mathbf{k}^{\prime}-\mathbf{k}_{\mathbf{r}}\right) \cdot \mathbf{r}}\left(\frac{\left|\mathbf{k}^{\prime}-\mathbf{k}_{\mathbf{r}}\right|^{2}}{2\left(\mathbf{k}^{\prime}-\mathbf{k}_{\mathbf{r}}\right) \cdot \mathbf{z}}\right) \Delta_{F}(\mathbf{r}) \times \\
\tilde{G}_{O}\left(\mathbf{k}^{\prime}\right) e^{2 \pi j \mathbf{k}^{\prime} \cdot \mathbf{r}^{\prime}} \mathrm{d}^{3} r d^{3} k^{\prime} .
\end{gathered}
$$

In this equation,

$$
\Delta_{F}(\mathbf{r})=4 \pi j R W\left(r_{x}, r_{y}\right) \delta\left(r_{z}-s\left(r_{x}, r_{y}\right)\right),
$$

is the function that we refer to as the foil model of the surface, where the surface profile (height) is defined by $s\left(r_{x}, r_{y}\right), R$ is the Fresnel amplitude reflection coefficient at normal incidence, $E_{r}$ and $\mathbf{k}_{\mathbf{r}}$ are the amplitude and the $\mathbf{k}$ vector of the incident wave, and $W\left(r_{x}, r_{y}\right)$ is a window function, which is different from zero only for the illuminated region of the surface. Fourier transformation gives the spectral density of the portion of the scattered field that is collected by the aperture of a far-field optical instrument such that,

$$
\tilde{E}_{m}(\mathbf{k})=E_{r} \tilde{G}_{O}(\mathbf{k})\left(\frac{\left|\mathbf{k}-\mathbf{k}_{\mathbf{r}}\right|^{2}}{2\left(\mathbf{k}-\mathbf{k}_{\mathbf{r}}\right) \cdot \mathbf{z}}\right) \int e^{-2 \pi j\left(\boldsymbol{k}-\mathbf{k}_{\mathbf{r}}\right) \cdot \mathbf{r}} \Delta_{F}(\mathbf{r}) \mathrm{d}^{3} r .
$$

In order to analyse the 3D image recorded by a FVM, we now decompose the surface profile function, $s\left(r_{x}, r_{y}\right)$, into two components; a smooth or specular component, $s_{S}\left(r_{x}, r_{y}\right)$, that represents the surface form and varies slowly on the scale of a wavelength; and a diffuse component, $s_{D}\left(r_{x}, r_{y}\right)$, that is a small perturbation that varies quickly on the scale of a wavelength, and results in diffusely scattered light, such that,

$$
s\left(r_{x}, r_{y}\right)=s_{S}\left(r_{x}, r_{y}\right)+s_{D}\left(r_{x}, r_{y}\right)
$$

Substituting we have,

$$
\begin{gathered}
\tilde{E}_{m}(\mathbf{k})=E_{r} \tilde{G}_{O}(\mathbf{k})\left(\frac{\left|\mathbf{k}-\mathbf{k}_{\mathbf{r}}\right|^{2}}{2\left(\mathbf{k}-\mathbf{k}_{\mathbf{r}}\right) \cdot \mathbf{z}}\right) \times \\
\int 4 \pi j R W\left(r_{x}, r_{y}\right) e^{-2 \pi j\left[\left(\mathbf{k}-\mathbf{k}_{\mathbf{r}}\right) \cdot \mathbf{r}_{x}+\left(\mathbf{k}-\mathbf{k}_{\mathbf{r}}\right) \cdot \mathbf{r}_{y}\right]} \times \\
e^{-2 \pi j\left(\mathbf{k}-\mathbf{k}_{\mathbf{r}}\right) \cdot \mathbf{z}\left[s_{S}\left(r_{x}, r_{y}\right)+s_{D}\left(r_{x}, r_{y}\right)\right]} \mathrm{d} r_{x} \mathrm{~d} r_{y}
\end{gathered}
$$

If the amplitude of the diffuse component is small, such that $2 \pi j\left(\mathbf{k}-\mathbf{k}_{\mathbf{r}}\right) \cdot \mathbf{z} s_{D}\left(r_{x}, r_{y}\right) \ll 1 \quad, \quad$ we can write, $e^{-2 \pi j\left(\mathbf{k}-\mathbf{k}_{\mathbf{r}}\right) \cdot \mathbf{z}\left[s_{D}\left(r_{x}, r_{y}\right)\right]} \approx\left[1-2 \pi j\left(\mathbf{k}-\mathbf{k}_{\mathbf{r}}\right) \cdot \mathbf{z} s_{D}\left(r_{x}, r_{y}\right)\right]$ and the measured field can be written,

$$
\begin{gathered}
\tilde{E}_{m}(\mathbf{k})=E_{r} \tilde{G}_{O}(\mathbf{k})\left(\frac{\left|\mathbf{k}-\mathbf{k}_{\mathbf{r}}\right|^{2}}{2\left(\mathbf{k}-\mathbf{k}_{\mathbf{r}}\right) \cdot \mathbf{z}}\right) \int e^{-2 \pi j\left(\mathbf{k}-\mathbf{k}_{\mathbf{r}}\right) \cdot \mathbf{r}} \times \\
{\left[1-2 \pi j\left(\mathbf{k}-\mathbf{k}_{\mathbf{r}}\right) \cdot \mathbf{z} s_{D}\left(r_{x}, r_{y}\right)\right] \Delta_{F S}(\mathbf{r}) \mathrm{d}^{3} r,}
\end{gathered}
$$


where $\Delta_{F S}(\mathbf{r})=4 \pi j R W\left(r_{x}, r_{y}\right) \delta\left(r_{z}-s_{S}\left(r_{x}, r_{y}\right)\right)$ is a foil model of the smooth component of the surface form. Writing $\tilde{E}_{m}(\mathbf{k})=\tilde{E}_{S}(\mathbf{k})+\tilde{E}_{D}(\mathbf{k})$, the measured field can be split into specular and diffusely scattered components given by,

$$
\begin{gathered}
\widetilde{E}_{S}(\mathbf{k})=E_{r} \tilde{G}_{O}(\mathbf{k})\left(\frac{\left|\mathbf{k}-\mathbf{k}_{\mathbf{r}}\right|^{2}}{2\left(\mathbf{k}-\mathbf{k}_{\mathbf{r}}\right) \cdot \mathbf{z}}\right) \int e^{-2 \pi j\left(\mathbf{k}-\mathbf{k}_{\mathbf{r}}\right) \cdot \mathbf{r}} \Delta_{F S}(\mathbf{r}) \mathrm{d}^{3} r . \\
\tilde{E}_{D}(\mathbf{k})=-\pi j E_{r} \tilde{G}_{O}(\mathbf{k})\left|\mathbf{k}-\mathbf{k}_{\mathbf{r}}\right|^{2} \times \\
\int e^{-2 \pi j\left(\mathbf{k}-\mathbf{k}_{\mathbf{r}}\right) \cdot \mathbf{r}} S_{D}\left(r_{x}, r_{y}\right) \Delta_{F S}(\mathbf{r}) \mathrm{d}^{3} r .
\end{gathered}
$$

It is clear that the specular and diffuse components of the measured scattered field are linear functions of the smooth surface form $\Delta_{F S}(\mathbf{r})$. It is noted, however, that a FVM records the scattered intensity of the collected field and this implies that the corresponding spectra are mixed according to the autocorrelation theorem [6], such that,

$$
\tilde{I}_{m}(\mathbf{k})=\int \tilde{E}_{m}\left(\mathbf{k}^{\prime}\right) \tilde{E}_{m}^{*}\left(\mathbf{k}^{\prime}-\mathbf{k}\right) \mathrm{d}^{3} k^{\prime}
$$

Finally we note that a FVM generally illuminates the sample either through the objective or with additional extra-aperture illumination. If the numerical aperture of the illuminating system is $N_{I}$, then the $3 \mathrm{D}$, incoherent image, $\tilde{O}_{m}(\mathbf{k})$, can be written in $\mathrm{k}$-space as,

$$
\tilde{O}_{m}(\mathbf{k})=\iint \tilde{E}_{m}\left(\mathbf{k}^{\prime}\right) \tilde{E}_{m}^{*}\left(\mathbf{k}^{\prime}-\mathbf{k}\right) d^{3} k^{\prime}\left|\tilde{G}_{I}\left(-\mathbf{k}_{r}\right)\right| \mathrm{d}^{3} k_{r},
$$

where

$$
\tilde{G}_{I}(\mathbf{k})=\frac{j}{4 \pi k_{0}} \delta\left(|\mathbf{k}|-k_{0}\right) \operatorname{step}\left(\frac{\mathbf{k} . \widehat{\mathbf{o}}}{k_{0}}-\sqrt{1-N_{I}^{2}}\right) .
$$

Remembering that the scattered field is the sum of specular and diffuse components, $\tilde{E}_{m}(\mathbf{k})=\tilde{E}_{S}(\mathbf{k})+\tilde{E}_{D}(\mathbf{k})$, the output can be split into terms that represent the interaction of these components, such that,

$$
\tilde{O}_{m}(\mathbf{k})=\tilde{O}_{S S}(\mathbf{k})+\tilde{O}_{S D}(\mathbf{k})+\tilde{O}_{D S}(\mathbf{k})+\tilde{O}_{D D}(\mathbf{k})
$$

where $\tilde{O}_{S D}(\mathbf{k})=\iint \tilde{E}_{S}\left(\mathbf{k}^{\prime}\right) \tilde{E}_{D}^{*}\left(\mathbf{k}^{\prime}-\mathbf{k}\right) d^{3} k^{\prime}\left|\tilde{G}_{I}\left(-\mathbf{k}_{\mathbf{r}}\right)\right| \mathrm{d}^{3} k_{r}$ etc. In order to understand the effect of mixing specular and diffusely scattered components it is useful to consider the form of each of these terms for the case of a scattering surface that has the form of an infinite plane. Without loss of generality we will assume that the normal to this plane, $\widehat{\mathbf{n}}$, is aligned with the $\mathbf{z}$-axis such that $\widehat{\mathbf{n}}=\mathbf{z}$. The analysis presented in APPENDIX B provides the form of the terms $\tilde{O}_{S S}(\mathbf{k}), \tilde{O}_{S D}(\mathbf{k}), \tilde{O}_{D S}(\mathbf{k})$ and $\tilde{O}_{D D}(\mathbf{k})$ in the $x z$ plane i.e. $\mathbf{k}=\left(k_{x}, 0, k_{z}\right)$. Accordingly the term,
$\tilde{O}_{S S}(\mathbf{k})$, that represents the self-interaction of the specular reflections, is given by,

$$
\begin{gathered}
\tilde{O}_{S S}(\mathbf{k})=\delta(\mathbf{k}) \frac{\left|E_{r} R\right|^{2}}{4 \pi k_{0}} \int \operatorname{step}\left(\frac{\mathbf{k}^{\mathrm{r}} \cdot \widehat{\mathbf{o}}}{k_{0}}-\sqrt{1-N_{O}^{2}}\right) \times \\
\operatorname{step}\left(\frac{-\mathbf{k}_{\mathbf{r}} \cdot \widehat{\mathbf{o}}}{k_{0}}-\sqrt{1-N_{I}^{2}}\right) \delta\left(\left|\mathbf{k}_{\mathbf{r}}\right|-k_{0}\right) \mathrm{d}^{3} k_{r}
\end{gathered}
$$

where $\mathbf{k}^{\mathrm{r}}=\mathbf{k}_{\mathbf{r}}-2\left(\mathbf{k}_{\mathbf{r}} \cdot \mathbf{z}\right) \mathbf{z}$ represents the reflected wave vector. It is clear from Eq. (22) that the term $\tilde{O}_{S S}(\mathbf{k})$ describes the zero frequency (DC) term characterised by a delta function at the origin and this corresponds to a uniform intensity in the output. The magnitude of this term depends on the angle of the instrument axis defined by $\widehat{\mathbf{o}}$ relative to the surface normal, $\mathbf{z}$. In practice this term is completely removed by the filter, $F_{F V M}(\mathbf{k})$, defined by Eq. (8).

Following the derivation in APPENDIX B, the terms $\tilde{O}_{S D}(\mathbf{k})$ and $\tilde{O}_{D S}(\mathbf{k})$ corresponding to interaction of the specular and diffusely scattered components are given by, the linear forms.

$$
\begin{aligned}
& \tilde{O}_{S D}(\mathbf{k})=\widetilde{\Delta}_{S}(\mathbf{k}) \widetilde{H}_{S D}(\mathbf{k}), \\
& \tilde{O}_{D S}(\mathbf{k})=\widetilde{\Delta}_{S}(\mathbf{k}) \widetilde{H}_{D S}(\mathbf{k}) .
\end{aligned}
$$

Here the transfer function $\widetilde{H}_{S D}(\mathbf{k})$, is given by,

$$
\widetilde{H}_{S D}(\mathbf{k})=\frac{\left|E_{r}\right|^{2} R^{*}}{16 \pi} \int \mathrm{d} k_{y}^{r} \frac{\left|\mathbf{k}-\left(\mathbf{2} \mathbf{k}_{0}^{\mathrm{r}} \cdot \mathbf{z}\right) \mathbf{z}\right|^{2}}{|\mathbf{k}| \sqrt{k_{0}^{2}-|\mathbf{k}|^{2} / 4-k_{y}^{\mathrm{r}}}} \times
$$

$$
\begin{gathered}
\operatorname{step}\left(\frac{k_{0 x}^{r} o_{x}+k_{0 Z}^{r} o_{z}-k_{x} o_{x}-k_{z} o_{z}}{k_{0}}-\sqrt{1-N_{O}^{2}}\right) \times \\
\operatorname{step}\left(\frac{k_{0 x}^{r} o_{x}+k_{0 z}^{r} o_{z}}{k_{0}}-\sqrt{1-N_{O}^{2}}\right) \operatorname{step}\left(\frac{-k_{0 z}^{r} o_{x}+k_{0 Z}^{r} o_{z}}{k_{0}}-\sqrt{1-N_{I}^{2}}\right),
\end{gathered}
$$

where,

$$
\begin{gathered}
k_{0 x}^{r}=\frac{1}{2}\left(k_{x}-\frac{k_{x} k_{z}}{\left|k_{x}\right||\mathbf{k}|} \sqrt{4 k_{N}^{2}-|\mathbf{k}|^{2}}\right), \\
k_{0 z}^{r}=\frac{1}{2}\left(k_{z}+\frac{\left|k_{x}\right|}{|\mathbf{|}|} \sqrt{4 k_{N}^{2}-|\mathbf{k}|^{2}}\right), \\
\text { and } k_{N}^{2}=k_{0}^{2}-k_{y}^{\mathrm{r}}{ }^{2} .
\end{gathered}
$$

Similarly the transfer function $H_{D S}(\boldsymbol{k})$ is given by,

$$
\widetilde{H}_{D S}(\mathbf{k})=-\frac{\left|E_{r}\right|^{2} R^{*}}{16 \pi} \int \mathrm{d} k_{y}^{m} \frac{\left|\mathbf{k}+\left(2 \mathbf{k}_{0}^{\mathrm{m}} \cdot \mathbf{z}\right) \mathbf{z}\right|^{2}}{|\mathbf{k}| \sqrt{k_{0}^{2}-|\mathbf{k}|^{2} / 4-k_{y}^{m^{2}}}} \times
$$




$$
\begin{gathered}
\operatorname{step}\left(\frac{k_{0 x}^{m} o_{x}+k_{0 Z}^{m} o_{z}+k_{x} o_{x}+k_{Z} o_{z}}{k_{0}}-\sqrt{1-N_{O}^{2}}\right) \times \\
\operatorname{step}\left(\frac{k_{0 x}^{m} o_{x}+k_{0 Z}^{m} o_{Z}}{k_{0}}-\sqrt{1-N_{O}^{2}}\right) \operatorname{step}\left(\frac{-k_{0 x}^{m} o_{x}+k_{0 Z}^{m} o_{Z}}{k_{0}}-\sqrt{1-N_{I}^{2}}\right),
\end{gathered}
$$

where,

$$
\begin{gathered}
k_{0 x}^{m}=\frac{1}{2}\left(-k_{x}-\frac{k_{x} k_{z}}{\left|k_{x}\right||\mathbf{k}|} \sqrt{4 k_{N}^{2}-|\mathbf{k}|^{2}}\right), \\
k_{0 z}^{m}=\frac{1}{2}\left(-k_{z}+\frac{\left|k_{x}\right|}{|\mathbf{k}|} \sqrt{4 k_{N}^{2}-|\mathbf{k}|^{2}}\right), \\
\text { and } k_{N}^{2}=k_{0}^{2}-k_{y}^{m^{2}} .
\end{gathered}
$$

It is clear that Eqs. (23) and (24) have the same linear form as Eq. (2) and correspond to a linear filtering operation applied to the foil representation of the surface form, $\widetilde{\Delta}_{S}(\mathbf{k})$. In contrast the incoherent transfer function, $\widetilde{H}_{B}(\mathbf{k})$ (derived in APPENDIX A), the filtering due to $\widetilde{H}_{S D}(\mathbf{k})$ and $\widetilde{H}_{D S}(\mathbf{k})$ is generally tilt dependent. Additionally, if there is no surface tilt such that, $\widehat{\mathbf{o}}=\mathbf{z}$ it is straightforward to show that the $\tilde{O}_{S D}(\mathbf{k})$ and $\tilde{O}_{D S}(\mathbf{k})$ terms cancel.

We will return to the significance of these findings later but for the moment let us move on to the term $\widetilde{O}_{D D}(\mathbf{k})$ corresponding to self-interaction of the diffusely scattered components which, following the derivation in APPENDIX B, is given by,

$$
\begin{gathered}
\tilde{O}_{D D}(\mathbf{k})=\frac{1}{16 \pi^{2}|\mathbf{k}|} \int \frac{\mathrm{d} k_{y}^{\prime}}{\sqrt{k_{0}^{2}-\frac{|\mathbf{k}|^{2}}{4}-k_{y}^{\prime 2}}} \times \\
\operatorname{step}\left(\frac{\mathbf{k}_{\mathbf{0}}^{\prime} \cdot \widehat{\mathbf{o}}}{k_{0}}-\sqrt{1-N_{O}^{2}}\right) \operatorname{step}\left(\frac{\left(\mathbf{k}_{\mathbf{0}}^{\prime}-\mathbf{k}\right) \cdot \widehat{\mathbf{o}}}{k_{0}}-\sqrt{1-N_{O}^{2}}\right) \times \\
\frac{\left|E_{r}\right|^{2} \pi}{4 k_{0}} \int \mathrm{d}^{3} k_{r}\left|\mathbf{k}_{\mathbf{0}}^{\prime}-\mathbf{k}_{\mathbf{r}}\right|^{2}\left|\mathbf{k}_{\mathbf{0}}^{\prime}-\mathbf{k}-\mathbf{k}_{\mathbf{r}}\right|^{2} \widetilde{\Delta}_{S}\left(\mathbf{k}_{\mathbf{0}}^{\prime}-\mathbf{k}_{\mathbf{r}}\right) \times \\
\widetilde{\Delta}_{S}^{*}\left(\mathbf{k}_{\mathbf{0}}^{\prime}-\mathbf{k}-\mathbf{k}_{\mathbf{r}}\right) \operatorname{step}\left(\frac{-\mathbf{k}_{\mathbf{r}} \cdot \widehat{\mathbf{o}}}{k_{0}}-\sqrt{1-N_{I}^{2}}\right) \delta\left(\left|\mathbf{k}_{\mathbf{r}}\right|-k_{0}\right),
\end{gathered}
$$

where the vector $\mathbf{k}_{\mathbf{0}}^{\prime}=\left(k_{0 x}^{\prime}, k_{y}^{\prime}, k_{0 z}^{\prime}\right)$ is with components:

$$
\begin{gathered}
k_{0 x}^{\prime}=\frac{1}{2}\left(k_{x}-\frac{k_{x} k_{z}}{\left|k_{x}\right||\mathbf{k}|} \sqrt{4 k_{N}^{2}-|\mathbf{k}|^{2}}\right), \\
k_{0 z}^{\prime}=\frac{1}{2}\left(k_{z}+\frac{\left|k_{x}\right|}{|\mathbf{k}|} \sqrt{4 k_{N}^{2}-|\mathbf{k}|^{2}}\right), \\
\text { and } k_{N}^{2}=k_{0}^{2}-k_{y}^{\prime 2} .
\end{gathered}
$$

It is important to realise that Eq. (33) does not have the linear form of Eq. (2). Comparison with Eq. (A12), however, shows that $\tilde{O}_{D D}(\mathbf{k})$ consists of two coupled integrals corresponding to the TF derived under the assumption of incoherent illumination, $\widetilde{H}_{B}(\mathbf{k})$, and the integral $I_{1}\left(\mathbf{k}, \mathbf{k}_{\mathbf{0}}^{\prime}\right)$, given by,

$$
\widetilde{H}_{B}(\mathbf{k})=\frac{1}{16 \pi^{2}|\mathbf{k}|} \int \frac{\mathrm{d} k_{y}^{\prime}}{\sqrt{k_{0}^{2}-\frac{|\mathbf{k}|^{2}}{4}-k_{y}^{\prime 2}}} \operatorname{step}\left(\frac{\mathbf{k}_{\mathbf{0}}^{\prime} \cdot \widehat{\mathbf{o}}}{k_{0}}-\sqrt{1-N_{O}^{2}}\right)
$$

$$
\times \operatorname{step}\left(\frac{\left(\mathbf{k}_{\mathbf{0}}^{\prime}-\mathbf{k}\right) . \widehat{\mathbf{o}}}{k_{0}}-\sqrt{1-N_{O}^{2}}\right)
$$

$$
\begin{gathered}
I_{1}\left(\mathbf{k}, \mathbf{k}_{\mathbf{0}}^{\prime}\right)=\frac{\left|E_{r}\right|^{2} \pi}{4 k_{0}} \int \mathrm{d}^{3} k_{r}\left|\mathbf{k}_{\mathbf{0}}^{\prime}-\mathbf{k}_{\mathbf{r}}\right|^{2}\left|\mathbf{k}_{\mathbf{0}}^{\prime}-\mathbf{k}-\mathbf{k}_{\mathbf{r}}\right|^{2} \times \\
\widetilde{\Delta}_{S}\left(\mathbf{k}_{\mathbf{0}}^{\prime}-\mathbf{k}_{\mathbf{r}}\right) \widetilde{\Delta}_{S}^{*}\left(\mathbf{k}_{\mathbf{0}}^{\prime}-\mathbf{k}-\mathbf{k}_{\mathbf{r}}\right) \times \\
\operatorname{step}\left(\frac{-\mathbf{k}_{\mathbf{r} . \widehat{\mathbf{o}}}}{k_{0}}-\sqrt{1-N_{I}^{2}}\right) \delta\left(\left|\mathbf{k}_{\mathbf{r}}\right|-k_{0}\right) .
\end{gathered}
$$

Considering $I_{1}\left(\mathbf{k}, \mathbf{k}_{\mathbf{0}}^{\prime}\right)$ first, it can be seen that this contribution introduces the non-linear self-mixing of the function, $\widetilde{\Delta}_{S}(\mathbf{k})$, that (in $k$-space) represents the roughened foil model of the surface (see APPENDIX B Eq. (B9)) given by,

$$
\widetilde{\Delta}_{S}(\mathbf{k})=\int e^{-2 \pi j \mathbf{k} \cdot \mathbf{r}} s_{D}\left(r_{x}, r_{y}\right) \Delta_{F S}(\mathbf{r}) \mathrm{d}^{3} r .
$$

For the case of a plane surface with surface normal in the $\mathrm{z}$ direction, it is noted however, that $\widetilde{\Delta}_{S}(\mathbf{k})$ is independent of $k_{z}$. If $s_{D}\left(r_{x}, r_{y}\right)$ is assumed to be white noise it follows that the product $\widetilde{\Delta}_{S}\left(\mathbf{k}_{\mathbf{0}}^{\prime}-\mathbf{k}_{\mathbf{r}}\right) \widetilde{\Delta}_{S}^{*}\left(\mathbf{k}_{\mathbf{0}}^{\prime}-\mathbf{k}-\mathbf{k}_{\mathbf{r}}\right)$ is essentially a random function of $k_{x}, k_{y}$ and the term $\left|\mathbf{k}_{\mathbf{0}}^{\prime}-\mathbf{k}_{\mathbf{r}}\right|^{2}\left|\mathbf{k}_{\mathbf{0}}^{\prime}-\mathbf{k}-\mathbf{k}_{\mathbf{r}}\right|^{2}$ represents a relatively slowly varying modulation. Making the substitution, $\mathbf{u}=\mathbf{k}_{\mathbf{0}}^{\prime}-\mathbf{k}_{\mathbf{r}}$, Eq. (38) can be simplified, such that,

$$
\begin{gathered}
I_{1}\left(\mathbf{k}, \mathbf{k}_{\mathbf{0}}^{\prime}\right)=-j\left|E_{r}\right|^{2} \pi^{2} \int \mathrm{d}^{3} u|\mathbf{u}|^{2}|\mathbf{u}-\mathbf{k}|^{2} \times \\
\widetilde{\Delta}_{S}(\mathbf{u}) \widetilde{\Delta}_{S}^{*}(\mathbf{u}-\mathbf{k}) \widetilde{G}_{I}\left(\mathbf{u}-\mathbf{k}_{\mathbf{0}}^{\prime}\right),
\end{gathered}
$$

where we have also used Eq. (20), $\tilde{G}_{I}(\mathbf{k})=\frac{j}{4 \pi k_{0}} \delta(|\mathbf{k}|-$ $\left.k_{0}\right) \operatorname{step}\left(\frac{\mathbf{k} . \widehat{\mathbf{o}}}{k_{0}}-\sqrt{1-N_{I}^{2}}\right)$. As mentioned previously, $\tilde{G}_{I}(\mathbf{k})$ defines the illumination aperture and can be considered to be a kernel that averages the terms, $|\mathbf{u}|^{2}|\mathbf{u}-\mathbf{k}|^{2} \widetilde{\Delta}_{S}(\mathbf{u}) \widetilde{\Delta}_{S}^{*}(\mathbf{u}-\mathbf{k})$ over a region defined in extent by the numerical aperture, $N_{I}$, and positioned according to the vector, $\mathbf{k}_{\mathbf{0}}^{\prime}=\left(\mathrm{k}_{0 \mathrm{x}}^{\prime}, \mathrm{k}_{\mathrm{y}}^{\prime}, \mathrm{k}_{0 \mathrm{z}}^{\prime}\right)$. From the previous argument it might be assumed that $I_{1}\left(\mathbf{k}, \mathbf{k}_{\mathbf{0}}^{\prime}\right)$ is independent of $k_{z}$, but this is not necessarily so. Through Eq. (34), $k_{0 x}^{\prime}=\frac{1}{2}\left(k_{x}-\frac{k_{x} k_{z}}{\left|k_{x}\right||\mathbf{k}|} \sqrt{4 k_{N}^{2}-|\mathbf{k}|^{2}}\right)$, the lateral position of $\tilde{G}_{I}\left(\mathbf{u}-\mathbf{k}_{\mathbf{0}}^{\prime}\right.$ ) (defined by $\mathrm{k}_{0 \mathrm{x}}^{\prime}$ and $\mathrm{k}_{\mathrm{y}}^{\prime}$ ) will depend strongly on $k_{z}$. Differentiating this relation at the point $\mathbf{k}=\left(k_{0} N_{O}, 0,0\right)$ (where the incoherent $\mathrm{TF}$ has maximum axial thickness) we find that $k_{0 x}^{\prime} \approx 2 k_{z} / N_{O}$ and it is straightforward to show that the half thickness at this point, $t \approx k_{0} N_{O}^{2} / 2$. Further from Eq. (20), it is straightforward to show that the lateral half width of $\tilde{G}_{I}(\mathbf{k})$ in a plane normal to $\widehat{\mathbf{o}}$ is $w=k_{o} N_{I}$. Providing $\sqrt{k_{0 x}^{\prime 2}+\mathrm{k}_{\mathrm{y}}^{\prime 2}}<k_{o} N_{I}$ for all $k_{z}$ of interest, it is reasonable, therefore, to assume that $I_{1}\left(\mathbf{k}, \mathbf{k}_{\mathbf{0}}^{\prime}\right)$ is constant in the $\mathrm{z}$ direction (that defines the surface normal). In this case we require $\sqrt{k_{O}^{2} N_{O}^{2}+\mathrm{k}_{\mathrm{y}}^{\prime 2}}<k_{o} N_{I}$ or to a good approximation,

$$
N_{O}<N_{I}
$$


So, providing that the illumination aperture is at least that of the observation aperture, the $\tilde{O}_{D D}(\mathbf{k})$ term takes the form,

$$
\tilde{O}_{D D}(\mathbf{k})=\widetilde{H}_{B}(\mathbf{k}) \widetilde{\Delta}(\mathbf{k})
$$

where the surface is defined in k-space by a function $\widetilde{\Delta}(\mathbf{k})$ that is independent of $k_{z}$ and corresponds to a surface defined of the form is $\Delta(\mathbf{r})=I_{s}\left(r_{x}, r_{y}\right) \delta\left(r_{z}\right)$ where $I_{s}(\mathbf{r})$ is a (pseudo) random function. Comparison with Eqs. (2) and (3) shows that the $\tilde{O}_{D D}(\mathbf{k})$ term defined by Eq. (42) has an identical linear form to that derived under the assumption of incoherent scattering. Eq. (41) can be interpreted as the condition for the assumption of incoherent scattering to be valid.

In order to understand the behaviour of FVM it is necessary to consider the relative importance of the terms $\tilde{O}_{S S}(\mathbf{k}), \tilde{O}_{S D}(\mathbf{k})$, $\tilde{O}_{D S}(\mathbf{k})$ and $\tilde{O}_{D D}(\mathbf{k})$ that, in k-space, characterise a through focus, 3D image of a rough surface as follows.

\section{DISCUSSION}

The analysis presented in the previous section provides a kspace representation of the 3D image formed by a FVM as it scans through focus. The analysis was based on a foil model of surface scattering where the surface profile function, $s\left(r_{x}, r_{y}\right)$ was decomposed into a form component, $s_{S}\left(r_{x}, r_{y}\right)$, that is slowly varying and resolved by the instrument, together with a small unresolved perturbation, $s_{D}\left(r_{x}, r_{y}\right)$. Using this approach the scattered field was decomposed into specular and diffusely scattered components and the image stack output by a FVM was characterised by the terms $\widetilde{O}_{S S}(\mathbf{k}), \tilde{O}_{S D}(\mathbf{k}), \tilde{O}_{D S}(\mathbf{k})$ and $\tilde{O}_{D D}(\mathbf{k})$ corresponding to the combination of these components in the output images.

The relative size of each term depends on the amplitude of $s_{D}\left(r_{x}, r_{y}\right)$. It is clear that if the surface roughness is small such that $s_{D}\left(r_{x}, r_{y}\right) \ll \lambda, \tilde{O}_{S D}(\mathbf{k}), \tilde{O}_{D S}(\mathbf{k})$ and $\tilde{O}_{D D}(\mathbf{k})$ are negligible and the term, $\tilde{O}_{S S}(\mathbf{k})$, that represents the self-mixing of the specular reflection dominates. It is clear from Eq. (22), however, that this term is a scaled delta function at the origin and therefore merely represents the uniform background intensity that is removed by the filter $F_{F V M}(\mathbf{k})$ that is applied during analysis process as described in Section 1.

As $s_{D}\left(r_{x}, r_{y}\right)$ increases the terms $\tilde{O}_{S D}(\mathbf{k})$ and $\tilde{O}_{D S}(\mathbf{k})$ appear in the output. These represent the interaction of the diffusely scattered components with the specular reflections. The terms can be written as linear filtering operations that are characterised by the transfer functions $\widetilde{H}_{S D}(\mathbf{k})$ and $\widetilde{H}_{D S}(\mathbf{k})$ defined in closed form by Eqs. (25) and (29), and applied to a roughened foil model of the surface as defined in Eq. (39). In contrast with the incoherent transfer function, $\widetilde{H}_{B}(\mathbf{k})$, derived in Section $1, \widetilde{H}_{S D}(\mathbf{k})$ and $\widetilde{H}_{D S}(\mathbf{k})$ depend on the tilt of the sample relative to the optical axis of the instrument and relative numerical apertures, $N_{I}$ and $N_{O}$, of the illumination and observation optics respectively. If the surface normal coincides with the optical axis of a typical instrument using through the lens illumination such that $N_{O}=N_{I}$ it is straightforward to show that the $\tilde{O}_{S D}(\mathbf{k})$ and $\tilde{O}_{D S}(\mathbf{k})$ terms completely cancel. If however, the surface is tilted, this is not the case and the transfer function $\widetilde{H}_{S D}(\mathbf{k})+\widetilde{H}_{D S}(\mathbf{k})$ is asymmetric. Figure 3 shows sections in the plane $k_{y}=0$ for the combined TFs
$\left(\widetilde{H}_{S D}(\mathbf{k})+\widetilde{H}_{D S}(\mathbf{k})\right)$ and corresponding PSFs as a function of angle for a quasi-monochromatic FVM with $N_{O}=N_{I}=0.5$.

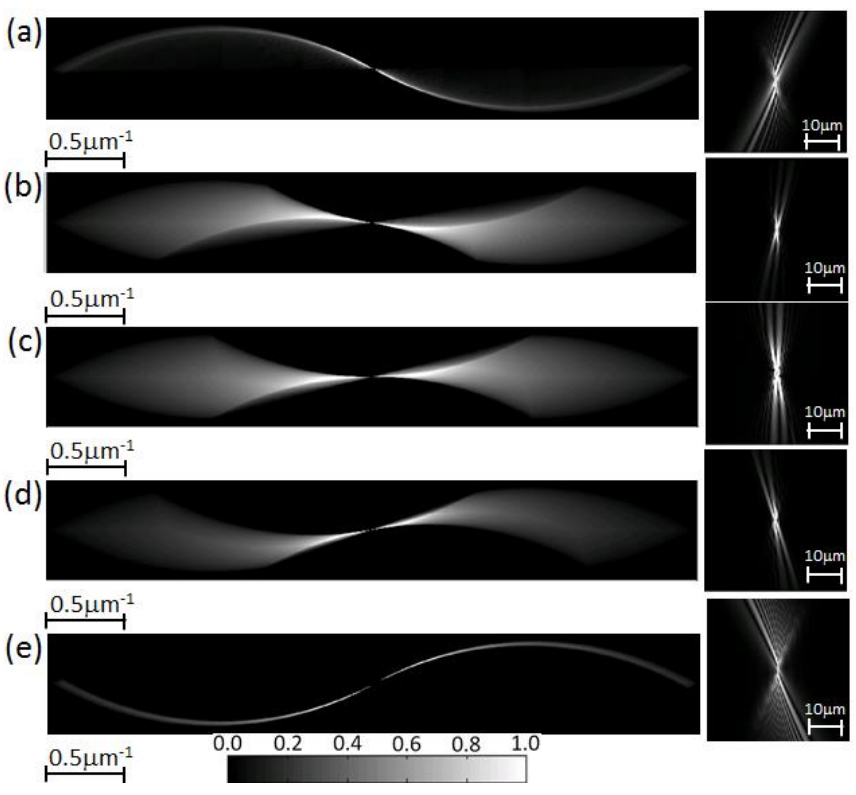

Fig. 3. Normalised sections in the plane $k_{y}=0$ for the combined TFs $\left(\widetilde{H}_{S D}(\mathbf{k})+\widetilde{H}_{D S}(\mathbf{k})\right)$ (left) and corresponding PSFs as a function of angle (right) for a quasi-monochromatic FVM with $N_{O}=N_{I}=0.5$ with surface tilt a) $1^{0}$, b) $10^{\circ}$, c) $15^{\circ}$, d) $25^{\circ}$, e) $29^{\circ}$

It is clear from figure 3 that the tilt dependence of the TF/PSF is most visible when the surface normal is tilted at $29^{\circ}$ from the optical axis of the instrument. At this angles only a small fraction of the specular reflections of the wave vector components transmitted by illumination aperture are collected by the observation aperture. Remarkably a similar result is observed at $1^{0}$ where $\widetilde{H}_{S D}(\mathbf{k})+\widetilde{H}_{D S}(\mathbf{k})$ cancel over the majority but not all of k-space. Interestingly $\widetilde{H}_{S D}(\mathbf{k})$ and $\widetilde{H}_{D S}(\mathbf{k})$ also cancel for the case of a tilted surface if the illumination aperture is increased.

(a)

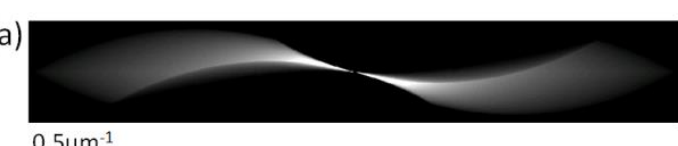

(b)
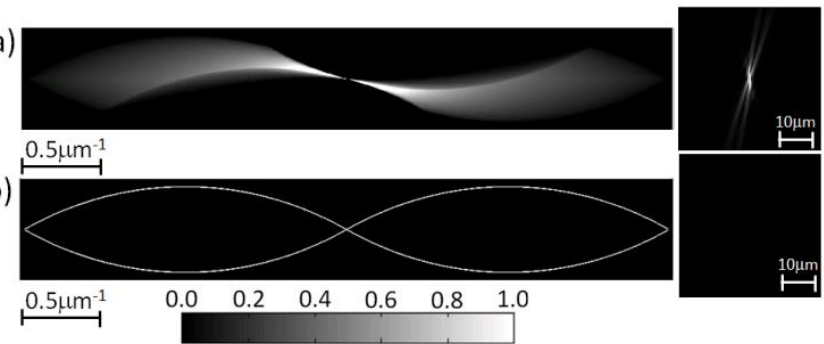

Fig 4. Normalised sections in the plane $k_{y}=0$ for the combined TFs $\left(\widetilde{H}_{S D}(\mathbf{k})+\widetilde{H}_{D S}(\mathbf{k})\right)$ (left) and corresponding PSFs (right) for a quasimonochromatic FVM with surface tilt of $15^{\circ}$ and $N_{O}=0.5$; a) $N_{I}=\sin 45^{\circ}$ and b) $N_{I}=\sin 60^{\circ}$.

Figure 4 shows the TF and PSF for the case of surface tilt of $15^{\circ}$ when the illumination aperture is increased to a) $N_{I}=\sin 45^{\circ}$ and b) $N_{I}=\sin 60^{\circ}$. It is clear that when $N_{I}=\sin 60$ the $\widetilde{H}_{S D}(\mathbf{k})$ and $\widetilde{H}_{D S}(\mathbf{k})$ terms cancel once again (the outline has been added to indicate the position of the incoherent TF). It is straightforward to show that this condition is satisfied if,

$$
\sin ^{-1} N_{I}>\sin ^{-1} N_{O}+2 \sin ^{-1}(\widehat{\mathbf{n}} . \widehat{\mathbf{o}})
$$


where $\widehat{\boldsymbol{n}}$ and $\widehat{\boldsymbol{o}}$ are unit vectors in the direction of the surface normal and the optical axis respectively.

The final term, $\tilde{O}_{D D}(\mathbf{k})$, is the dominant term and the only significant term in the output image of the FVM if i) the $\widetilde{O}_{S D}(\mathbf{k})$ and $\tilde{O}_{D S}(\mathbf{k})$ terms completely cancel as previously discussed or ii) the surface is tilted such that no specular reflection is collected by the observation aperture or iii) the surface is sufficiently rough that there is no significant specular deflection. All of these conditions are possible in practice. The analysis presented in the previous section shows that providing the illumination aperture is sufficient this output can be considered as a linear filtering operation characterised by the incoherent transfer function, $\widetilde{H}_{B}(\mathbf{k})$, applied to a foil model of the surface with a modulation that depends on the both the surface roughness and the numerical apertures, $N_{I}$ and $N_{O}$, of the illumination and observation optics. A sufficient condition for this is,

$$
N_{I}>N_{O}
$$

If this condition is not fully satisfied it can be shown that the axial resolution of the instrument will progressively decrease. Outputs $\tilde{O}_{D D}(\mathbf{k})$ together with sections through the corresponding surface images calculated using the filter specified are presented in figure 5 for different illumination apertures, $N_{I}$. In this figure the phase of $\tilde{O}_{D D}(\mathbf{k})$ that is plotted while the surface images are the real values. It can be seen that as $N_{I}$ decreases the axial resolution decreases as expected.

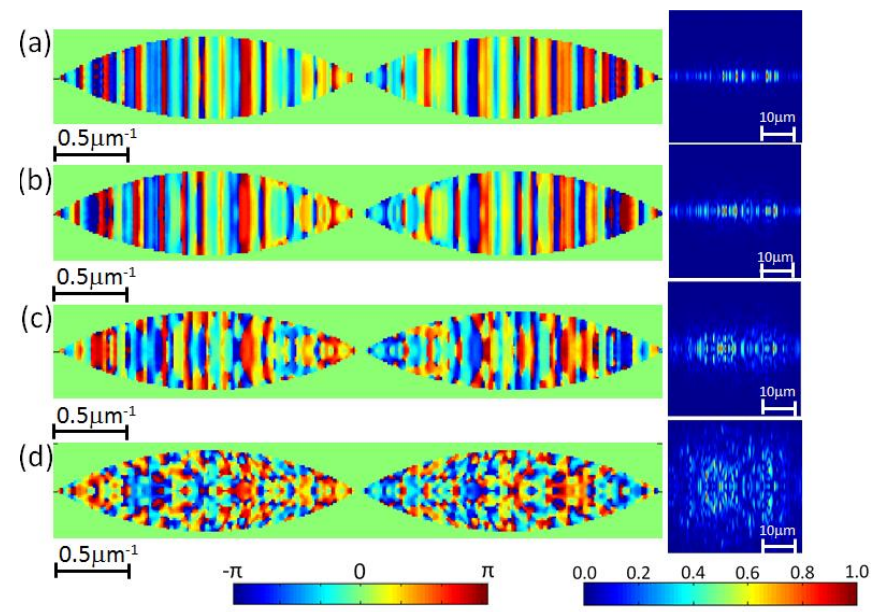

Fig. 5. Phase of $\tilde{O}_{D D}(\mathbf{k})$ (left) together with sections through normalised surface images for a quasi-monochromatic FVM (right) with surface tilt of $0^{\circ}$ and $N_{O}=0.5$; a) $N_{I}=0.5$, b) $N_{I}=0.25$, c) $N_{I}=0.1$ and d) $N_{I}=0.01$.

\section{CONCLUSIONS}

In this paper we have considered 3D image formation using the focus variation microscope (FVM) as a linear filtering process. In this way a FVM can be characterised in the frequency domain (k-space) by its transfer function (TF) or equivalently, in the space domain by its point spread function (PSF). In essence these functions define the 3D resolution of the instrument and its ability to measure surface form. With the assumption of incoherent scattering, it is shown that the imaging process takes a linear form and a closed form solution of the TF is derived for the case of a quasi-monochromatic instrument. It is shown that the TF and PSF are a well-defined function of the numerical aperture of the observation optics.

It is argued however, that, for the case of micro-scale roughness and illumination optics of restricted numerical aperture, the assumption of incoherence cannot be justified and more rigorous analysis is required. Using a foil model of surface scattering that we have discussed elsewhere [5], the scattered fields and 3D output of a FVM was calculated. An important aspect of this analysis is the separation of the specular and diffusely scattered components of the field collected by the instrument. In this way the TF was calculated for the case of an infinite plane surface of well-defined roughness. It is shown that in general, the TF depends on surface tilt and is attributed to the mixing of specular and diffusely reflected components.

The tilt dependence depends strongly on the numerical aperture of the illumination optics. For the case of illumination through the imaging optics (as is frequently the case in practice) the TF is modified most significantly when the tilt angle approaches the acceptance angle of imaging/illumination aperture. It is noted, however, that tilt dependence is avoided if the illumination aperture is sufficient to ensure that a specular reflection of the source is collected across the entire observation aperture. In general, this requires that the illumination aperture is significantly larger than that of the observation optics (Eq. (43)). Finally it is shown that the TF derived using the foil model of surface scattering is reduced to that derived with the assumption of incoherent scattering providing that the numerical aperture of the illumination optics is at least as large as the observation optics (Eq. (44)). If this is not satisfied the axial resolution of the instrument is decreased proportionately.

It should be emphasised however, that many commercially available focus variation microscopes provide additional resources to prevent or mitigate the described effects. In addition to increasing the illumination aperture (which can be realized by using ring lights that can be mounted on the objective), polarization filters can be introduced to attenuate or block the specular light components and hence reduce or eliminate the tilt dependent response.

Finally it should be mentioned that focus variation microscopes are typically applied to surfaces which have roughness that lies above the range where the tilt dependent response is observed (i.e. $\mathrm{Ra} \gg>50 \mathrm{~nm}$ ). In addition the use of polychromatic light and optical aberrations that are present in real systems are expected to have significant effect on both the resolution and the tilt dependence of the transfer function. Accordingly, further work is planned to compare experimental observations with the predictions of the theory presented here.

\section{Acknowledgment.}

The Authors wish to acknowledge the help of Prof. Nikolay Mitov Nikolov of the Bulgarian Academy of Sciences, Sofia Bulgaria and are grateful to the European Union for supporting the work under the grant IND59 "Microparts".

\section{References}


1. R. K. Leach, ed., Optical Measurement of Surface Topography (Springer Berlin Heidelberg, 2011)

2. F. Hiersemenzel, J. Petzing, R. Leach, F. Helmli, F. Singh, "Areal texture and angle measurements of tilted surfaces using focus variation methods," in 3rd Int. Conf. Surface Metrology, Annecy, France, 2012, pp. 1-5.

3. J. Coupland and N. Nikolaev, "The influence of tilt on surface roughness measurement using the focus variation microscope," Proceedings of the $15^{\text {th }}$ International Conference of the European Society for Precision Engineering and Nanotechnology, EUSPEN, (2015), pp.155-156 ISBN: 9780956679079.

4. J. M. Coupland and J. Lobera, "Holography, tomography and 3D microscopy as linear filtering operations", Meas. Sci. Technol. 19, 074012 (2008).

5. J. Coupland, R. Mandal, K. Palodhi and R. Leach, "Coherence scanning interferometry: linear theory of surface measurement," Appl. Optics 52, 3662-3670 (2013).

6. J. W. Goodman, Introduction to Fourier Optics (New York: McGrawHill, 1996).

7. C. J. R. Sheppard, "Scanning Confocal Microscopy" in Encyclopedia of Optical Engineering (Marcel Dekker, Inc. New York 2003) 25252544.

8. V. Lauer, "New approach to optical diffraction tomography yielding a vector equation of diffraction tomography and a novel tomographic microscope," J. Microsc. 205, 165-176 (2002).

9. M. Niederöst, J. Niederöst, J. Scucka, "Automatic 3D reconstruction and visualization of microscopic objects from a monoscopic multifocus image sequence," International Archives of the Photogarmmetry, Remote Sensing and Spatial Information Sciences XXXIV 5/W10, 1682-1777 (2003)

10. S. K. Nayar and Y. Nakagawa, "Shape from focus," IEEE Transactions on Pattern Analysis and Machine Intelligence, 16 , 824-831 (1994).

11. L. Hörmander, in The Analysis of Linear Partial Differential Operators I Distribution Theory and Fourier Analysys, (Springer Verlag Berlin Heidelberg New York Tokyo 1983) Chapter 6.3.

\section{APPENDIX A}

In the following we calculate an analytic expression for the transfer function (TF) of an axis-symmetric optical system, defined by,

$$
\widetilde{H}_{B}(\mathbf{k})=\int \tilde{G}_{O}\left(\mathbf{k}^{\prime}\right) \tilde{G}_{O}^{*}\left(\mathbf{k}^{\prime}-\mathbf{k}\right) \mathrm{d}^{3} k^{\prime},
$$

where

$$
\tilde{G}_{O}(\mathbf{k})=\frac{j}{4 \pi k_{0}} \delta\left(|\mathbf{k}|-k_{0}\right) \operatorname{step}\left(\frac{\mathbf{k} . \widehat{\mathbf{o}}}{k_{0}}-\sqrt{1-N_{O}^{2}}\right) .
$$

Substituting Eq. (A2) in (A1) we have

$$
\begin{gathered}
\widetilde{H}_{B}(\mathbf{k})=\frac{1}{16 \pi^{2} k_{0}^{2}} \int \operatorname{step}\left(\frac{\mathbf{k}^{\prime} . \widehat{\mathbf{o}}}{k_{0}}-\sqrt{1-N_{O}^{2}}\right) \times \\
\operatorname{step}\left(\frac{\left(\mathbf{k}^{\prime}-\mathbf{k}\right) . \widehat{\mathbf{o}}}{k_{0}}-\sqrt{1-N_{O}^{2}}\right) \delta\left(\left|\mathbf{k}^{\prime}\right|-k_{0}\right) \delta\left(\left|\mathbf{k}^{\prime}-\mathbf{k}\right|-k_{0}\right) \mathrm{d}^{3} k^{\prime} .
\end{gathered}
$$

It is noted that each (1D) delta function in this equation defines a spherical surface of radius $k_{0}$. Since the centres of the spheres are separated by a distance $|\mathbf{k}|$, their product defines a circle that is the intersection of the spherical surfaces. It is also noted that due to the finite aperture of the instrument, only k-vectors that are represented by a small "cap-like" portion of the sphere are collected according to the step functions in Eq. (A3). Because of this, the integrand represents a circular arc in $k$ space as shown in figure A1.

In order to evaluate integral we will use following transformation [11],

$$
\begin{gathered}
\delta\left(y_{1}\right) \delta\left(y_{2}\right) \ldots \delta\left(y_{n}\right)=\frac{1}{|\operatorname{det}(J)|} \delta\left(x_{1}-a_{1}\right) \times \\
\delta\left(x_{2}-a_{2}\right) \ldots \delta\left(x_{n}-a_{n}\right),
\end{gathered}
$$

where $\quad y_{1}=y_{1}\left(x_{1}, x_{2}, \ldots x_{n}\right) ; y_{2}=y_{2}\left(x_{1}, x_{2}, \ldots x_{n}\right)$, $\ldots, y_{n}=y_{n}\left(x_{1}, x_{2}, \ldots x_{n}\right)$ with roots $a_{1}, a_{2} \ldots a_{n}$ (such that $y_{i}\left(a_{1}, a_{2}, \ldots a_{n}\right)=0$ ) and $\boldsymbol{J}$ is the Jacobian of the transformation from $y_{1}, y_{2} \ldots y_{n}$ to $x_{1}, x_{2}, \ldots x_{n}$ evaluated at points $x_{i}=a_{i}$, given by,

$\boldsymbol{J}_{i, j}=\left.\frac{\partial y_{i}}{\partial x_{i}}\right|_{x_{i}=a_{i}}$

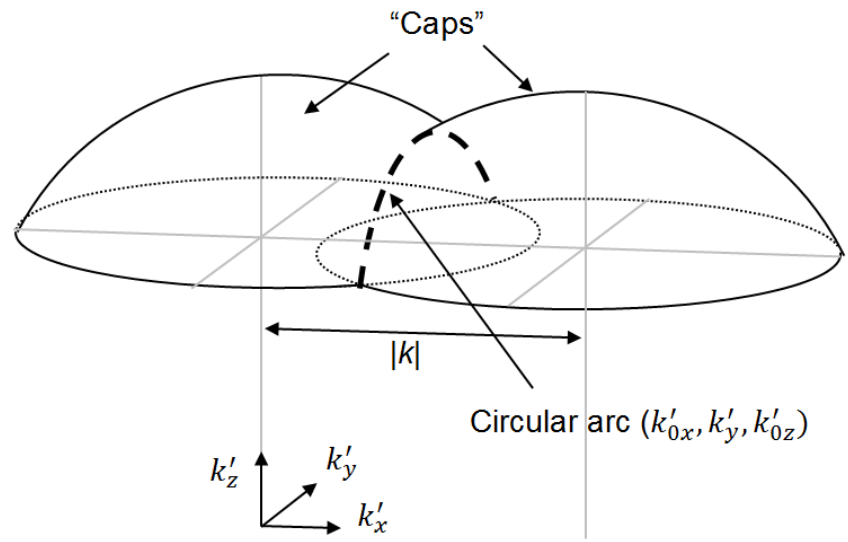

Fig。A1. Integration along a circular arc in k-space.

In this case, without loss of generality we consider a 2D slice in $\mathrm{k}$-space such that, $x_{1}=k_{x}^{\prime}, x_{2}=k_{z}^{\prime}$, and we have,

$$
\begin{gathered}
y_{1}=\left|\mathbf{k}^{\prime}\right|-k_{0}=\sqrt{{k_{x}^{\prime 2}+k_{y}^{\prime 2}+k_{z}^{\prime 2}}^{2}}-k_{0}, \\
y_{2}=\left|\mathbf{k}^{\prime}-\mathbf{k}\right|-k_{0}= \\
=\sqrt{\left(k_{x}^{\prime}-k_{x}\right)^{2}+\left(k_{y}^{\prime}-k_{y}\right)^{2}+\left(k_{z}^{\prime}-k_{z}\right)^{2}}-k_{0}
\end{gathered}
$$

The roots of these equations $a_{1}=k_{x 0}^{\prime}$ and $a_{2}=k_{z 0}^{\prime}$, define the intersection of the circular arc with the plane $k_{y}^{\prime}=k_{0 y}^{\prime}$. It is clear that in this case the transfer function is axis-symmetric and we need only to consider its form in any plane that passes through this axis. Accordingly in the plane $k_{y}=0$, it is found,

$$
\begin{aligned}
& k_{0 x}^{\prime}=\frac{1}{2}\left(k_{x}-\frac{k_{x} k_{z}}{\left|k_{x}\right||\mathbf{k}|} \sqrt{4 k_{N}^{2}-|\mathbf{k}|^{2}}\right), \\
& k_{0 z}^{\prime}=\frac{1}{2}\left(k_{z}+\frac{\left|k_{x}\right|}{|\mathbf{k}|} \sqrt{4 k_{N}^{2}-|\mathbf{k}|^{2}}\right),
\end{aligned}
$$


where $k_{N}^{2}=k_{0}^{2}-k_{y}^{\prime 2}$.

Performing the required differentiation and substituting into Eq. (A4) it is found,

$$
\begin{gathered}
\delta\left(\left|\mathbf{k}^{\prime}\right|-k_{0}\right) \delta\left(\left|\mathbf{k}^{\prime}-\mathbf{k}\right|-k_{0}\right)=\frac{k_{0}^{2}}{\left|k_{0 z}^{\prime} k_{x}-k_{0 x}^{\prime} k_{z}\right|} \delta\left(k_{x}^{\prime}-k_{0 x}^{\prime}\right) \times \\
\delta\left(k_{z}^{\prime}-k_{0 z}^{\prime}\right)
\end{gathered}
$$

Using this transformation, the 3D integral in Eq. (A3) reduces to a one dimensional integral given by,

$$
\begin{aligned}
& \widetilde{H}_{B}(\mathbf{k})=\frac{1}{16 \pi^{2} k_{0}^{2}} \int \operatorname{step}\left(\frac{\mathbf{k}^{\prime} \cdot \widehat{\mathbf{o}}}{k_{0}}-\sqrt{1-N_{O}^{2}}\right) \times \\
& \operatorname{step}\left(\frac{\left(\mathbf{k}^{\prime}-\mathbf{k}\right) \cdot \widehat{\mathbf{o}}}{k_{0}}-\sqrt{1-N_{O}^{2}}\right) \frac{k_{0}^{2}}{\left|k_{0 z}^{\prime} k_{x}-k_{0 x}^{\prime} k_{z}\right|} \times \\
& \delta\left(k_{x}^{\prime}-k_{0 x}^{\prime}\right) \delta\left(k_{z}^{\prime}-k_{0 z}^{\prime}\right) \mathrm{d}^{3} k^{\prime} .
\end{aligned}
$$

Using Eqs. (A7) and (A8) once again the term $\left|k_{0 z}^{\prime} k_{x}-k_{0 x}^{\prime} k_{z}\right|$ in this integral can be written,

$$
\left|k_{0 z}^{\prime} k_{x}-k_{0 x}^{\prime} k_{z}\right|=|\mathbf{k}| \sqrt{k_{0}^{2}-|\mathbf{k}|^{2} / 4-k_{y}^{\prime 2}} .
$$

And,

$$
\begin{aligned}
& \widetilde{H}_{B}(\mathbf{k})=\frac{1}{16 \pi^{2}|\mathbf{k}|} \int \operatorname{step}\left(\frac{\mathbf{k}_{0}^{\prime} \cdot \widehat{\mathbf{o}}}{k_{0}}-\sqrt{1-N_{O}^{2}}\right) \times \\
& \operatorname{step}\left(\frac{\left(\mathbf{k}_{0}^{\prime}-\mathbf{k}\right) \cdot \widehat{\mathbf{o}}}{k_{0}}-\sqrt{1-N_{O}^{2}}\right) \frac{1}{\sqrt{k_{0}^{2}-|\mathbf{k}|^{2} / 4-k_{y}^{\prime 2}}} \mathrm{~d} k_{y}^{\prime},
\end{aligned}
$$

where $\mathbf{k}_{0}^{\prime}=\left(k_{0 x}^{\prime}, k_{y}^{\prime}, k_{0 z}^{\prime}\right)$.

Now without loss of generality we assume that the optical axis of the instrument is aligned to the $\mathrm{z}$ axis such that, $\widehat{\mathbf{o}}=\mathbf{z}$. As mentioned previously the step() functions in this expression represent "cap-like" regions of the spherical surfaces (see figure A1) and effectively define the limits of integration. Analysis of the arguments of these functions provides $\left|k_{y}^{\prime}\right|<$ $B$ where $\mathrm{B}$ is given by,

$$
B=\sqrt{\left(k_{0}^{2}-|\mathbf{k}|^{2} / 4\right)-\left[\frac{|\mathbf{k}|}{2\left|k_{z}\right|}\left(\left|k_{x}\right|+2 k_{0} \sqrt{1-N_{O}^{2}}\right)\right]^{2}} .
$$

Finally, noting that $\int 1 / \sqrt{a-x^{2}} \mathrm{~d} x=\tan ^{-1}\left(x / \sqrt{a-x^{2}}\right)+c$, the transfer function can be written,

$$
\widetilde{H}_{B}(\mathbf{k})=\frac{1}{8 \pi^{2}|\mathbf{k}|} \operatorname{step}\left(B^{2}\right) \tan ^{-1}\left(\frac{B}{\sqrt{k_{0}^{2}-|\mathbf{k}|^{2} / 4-B^{2}}}\right) \text {. }
$$

Eqs.(A13) and (A14) provide the cross-section $\left(k_{y}=0\right)$ corresponding to the transfer function defined by Eqs. (A1) and (A2) for the case of a symmetric optical instrument with its optical axis aligned with the $k_{z}$ direction. Noting once again that the transfer function is axis-symmetric, $k_{x}$ represents any radial component, and more generally we can write,

$$
B=\sqrt{\left(k_{0}^{2}-|\mathbf{k}|^{2} / 4\right)-\left[\frac{|\mathbf{k}|}{2 \sqrt{k_{x}^{2}+k_{y}^{2}}}\left(\left|k_{z}\right|+2 k_{0} \sqrt{1-N_{O}^{2}}\right)\right]^{2}} .
$$

Although Eqs. (A14) and (A15) are valid for any position $\mathbf{k}=\left(k_{x}, k_{y}, k_{z}\right)$ in $\mathrm{k}$-space, it is noted, that $|\mathbf{k}|$ in the denominator of Eq. (A14) results in a singularity of $\widetilde{H}_{B}(\mathbf{k})$ at the origin $|\mathbf{k}|=0$. We note however, that direct integration of Eq. (A1) at $\mathbf{k}=0$ gives,

$$
\widetilde{H}_{B}(0)=\frac{\delta(0)}{8 \pi}\left(1-\sqrt{1-N_{o}^{2}}\right),
$$

and provides the correct scaling of the zero-frequency component.

Eq. (A14) defines a closed form solution to the 3D $\mathrm{TF}$ that characterises the 3D images collected by a FVM with the assumption of incoherent scattering. It should be noted that this is a fundamental result that also corresponds to the TF of a coherent forward-scatter confocal microscope and the part of a the 3D image collected by a coherence scanning interferometer that is not modulated by interference fringes [5].

\section{APPENDIX B}

In this section we consider each of the terms in Eq. (21) that characterise the output image stack in FVM when incoherent scattering is not assumed. In the following the output is derived for the case of a roughened, infinite plane surface. The coordinate system is chosen such that the z-axis is normal to this surface such that $s_{S}\left(r_{x}, r_{y}\right)=0$. An axis-symmetric FVM with its optical axis defined by the unit vector $\widehat{\boldsymbol{o}}$ (that is not necessarily normal to the surface) is assumed. First the forms of the specular and diffuse components of the scattered fields are derived. Substituting the foil model $\Delta_{F S}(\mathbf{r})=4 \pi j R W\left(r_{x}, r_{y}\right) \delta\left(r_{z}-s_{S}\left(r_{x}, r_{y}\right)\right)$ with $s_{S}\left(r_{x}, r_{y}\right)=0 \quad$ in Eq. (16) the specular component, $\tilde{E}_{S}(\mathbf{k})$, corresponding to plane wave illumination, $\mathbf{k}_{\mathbf{r}}$, is given by,

$$
\begin{gathered}
\tilde{E}_{S}(\mathbf{k})=E_{r} \tilde{G}_{O}(\mathbf{k})\left(\frac{\left|\mathbf{k}-\mathbf{k}_{\mathbf{r}}\right|^{2}}{2\left(\mathbf{k}-\mathbf{k}_{\mathbf{r}}\right) \cdot \mathbf{z}}\right) \times \\
\int e^{-2 \pi j\left(\mathbf{k}-\mathbf{k}_{\mathbf{r}}\right) \cdot \mathbf{r}} 4 \pi j R W\left(r_{x}, r_{y}\right) \delta\left(r_{z}\right) \mathrm{d}^{3} r
\end{gathered}
$$

Assuming $W\left(r_{x}, r_{y}\right)=1$ (for an infinite plane) direct integration gives,

$$
\begin{aligned}
\tilde{E}_{S}(\mathbf{k})= & \frac{-R E_{r}}{k_{0}} \delta\left(|\mathbf{k}|-k_{0}\right) \operatorname{step}\left(\frac{\mathbf{k} \cdot \widehat{\mathbf{o}}}{k_{0}}-\sqrt{1-N_{O}^{2}}\right) \times \\
& \left(\frac{\left|\mathbf{k}-\mathbf{k}_{\mathbf{r}}\right|^{2}}{2\left(\mathbf{k}-\mathbf{k}_{\mathbf{r}}\right) \cdot \mathbf{z}}\right) \delta\left(k_{x}-k_{r x}\right) \delta\left(k_{y}-k_{r y}\right)
\end{aligned}
$$


where $\tilde{G}_{O}(\mathbf{k})$ has been substituted from Eq. (4). This expression is simplified by a change of the variables within the arguments of the delta functions using the transformation defined in Eq. (A4). Substituting $x_{1}=k_{x}, x_{2}=k_{y}, x_{3}=k_{z}$, $y_{1}=k_{x}-k_{r x}, y_{2}=k_{y}-k_{r y}, y_{3}=\sqrt{k_{x}^{2}+k_{y}^{2}+k_{z}^{2}}-k_{0}$ and the roots $a_{1}=k_{r x}, a_{2}=k_{r y}, a_{3}=-k_{r z}$, the absolute value of the determinant of the Jacobian of the transformation is,

$$
\left|\operatorname{det}\left(\boldsymbol{J}_{x=a}\right)\right|=\left|\operatorname{det}\left(\begin{array}{ccc}
1 & 0 & 0 \\
0 & 1 & 0 \\
\frac{k_{r x}}{k_{0}} & \frac{k_{r y}}{k_{0}} & \frac{-k_{r z}}{k_{0}}
\end{array}\right)\right|=\frac{\left|-k_{r z}\right|}{k_{0}}=\frac{\left|k_{r z}\right|}{k_{0}},
$$

whence it is found,

$$
\begin{aligned}
& \delta\left(k_{x}-k_{r x}\right) \delta\left(k_{y}-k_{r y}\right) \delta\left(|\mathbf{k}|-k_{0}\right)= \\
& =\frac{k_{0}}{\left|\mathbf{z} \cdot \mathbf{k}_{\mathbf{r}}\right|} \delta\left(\mathbf{k}-\left(\mathbf{k}_{\mathbf{r}}-\mathbf{2}\left(\mathbf{k}_{\mathbf{r}} \cdot \mathbf{z}\right) \mathbf{z}\right)\right) .
\end{aligned}
$$

Substituting Eq. (B4) into Eq. (B2) we find,

$$
\begin{aligned}
& \tilde{E}_{S}(\mathbf{k})=\frac{-R E_{r}}{k_{0}} \operatorname{step}\left(\frac{\mathbf{k} \cdot \widehat{\mathbf{o}}}{k_{0}}-\sqrt{1-N_{O}^{2}}\right) \times \\
& \left(\frac{\left|\mathbf{k}-\mathbf{k}_{\mathbf{r}}\right|^{2}}{2\left(\mathbf{k}-\mathbf{k}_{\mathbf{r}}\right) \cdot \mathbf{z}}\right) \frac{k_{0}}{\left|\mathbf{z} \cdot \boldsymbol{k}_{r}\right|} \delta\left(\mathbf{k}-\left(\mathbf{k}_{\mathbf{r}}-2\left(\mathbf{k}_{\mathbf{r}} \cdot \mathbf{z}\right) \mathbf{z}\right)\right) .
\end{aligned}
$$

Using the identity $f(x) \delta(x-a)=f(a) \delta(x-a)$ simplifying we find,

$$
\tilde{E}_{S}(\mathbf{k})=R E_{r} \operatorname{step}\left(\frac{\mathbf{k} \cdot \widehat{\mathbf{o}}}{k_{0}}-\sqrt{1-N_{O}^{2}}\right) \delta\left(\mathbf{k}-\left(\mathbf{k}_{\mathbf{r}}-\mathbf{2}\left(\mathbf{k}_{\mathbf{r}} \cdot \mathbf{z}\right) \mathbf{z}\right)\right)
$$

where it is noted that the term, $\mathbf{k}_{\mathbf{r}}-\mathbf{2}\left(\mathbf{k}_{\mathbf{r}}, \mathbf{z}\right) \mathbf{z}$ is simply the incident field with its component in the $\mathrm{z}$ direction reversed and consequently the scattered field described by Eq. (B6), simply describes the reflected plane wave that is either collected or rejected by the aperture of the instrument according to the step function. Although the term $\mathbf{k}_{\mathbf{r}}-\mathbf{2}\left(\mathbf{k}_{\mathbf{r}} \cdot \mathbf{z}\right) \mathbf{z}$ is clearly a function of the incident field wave vector, the term will appear frequently in the upcoming analysis and to simplify the notation we will denote the reflected term by $\mathbf{k}^{\mathrm{r}}$ as it arises such that,

$$
\mathbf{k}^{r}=\mathbf{k}_{\mathbf{r}}-2\left(\mathbf{k}_{\mathbf{r}} \cdot \mathbf{z}\right) \mathbf{z}
$$

Returning to the diffuse component, a similar analysis shows that the diffusely scattered field collected by the instrument, can be written in k-space as,

$$
\tilde{E}_{D}(\mathbf{k})=-E_{r} \pi j \tilde{G}_{O}(\mathbf{k})\left|\mathbf{k}-\mathbf{k}_{\mathbf{r}}\right|^{2} \widetilde{\Delta}_{S}\left(\mathbf{k}-\mathbf{k}_{\mathbf{r}}\right),
$$

where

$$
\widetilde{\Delta}_{S}(\mathbf{k})=\int e^{-2 \pi j \mathbf{k} \cdot \mathbf{r}} s_{D}\left(r_{x}, r_{y}\right) \Delta_{F S}(\mathbf{r}) \mathrm{d}^{3} r
$$

We are now in a position to derive each term in the FVM output $\tilde{O}_{m}(\mathbf{k})=\tilde{O}_{S S}(\mathbf{k})+\tilde{O}_{S D}(\mathbf{k})+\tilde{O}_{D S}(\mathbf{k})+\tilde{O}_{D D}(\mathbf{k})$.

\section{The $\tilde{O}_{S S}(\mathbf{k})$ term:}

First we derive the integral, $\tilde{I}_{S S}(\mathbf{k})=\int \tilde{E}_{S}\left(\mathbf{k}^{\prime}\right) \tilde{E}_{S}^{*}\left(\mathbf{k}^{\prime}-\mathbf{k}\right) \mathrm{d}^{3} k^{\prime}$, given by,

$$
\begin{gathered}
\tilde{I}_{S S}(\mathbf{k})=\int R E_{r} \operatorname{step}\left(\frac{\mathbf{k}^{\prime} \cdot \widehat{\mathbf{o}}}{k_{0}}-\sqrt{1-N_{O}^{2}}\right) \delta\left(\mathbf{k}^{\prime}-\mathbf{k}^{\mathrm{r}}\right) \times \\
R^{*} E_{r}^{*} \operatorname{step}\left(\frac{\left(\mathbf{k}^{\prime}-\mathbf{k}\right) \cdot \widehat{\mathbf{o}}}{k_{0}}-\sqrt{1-N_{O}^{2}}\right) \delta\left(\mathbf{k}^{\prime}-\mathbf{k}-\mathbf{k}^{\mathrm{r}}\right) \mathrm{d}^{3} k^{\prime} .
\end{gathered}
$$

Direct integration gives,

$$
\tilde{I}_{S S}(\mathbf{k})=\left|R E_{r}\right|^{2} \delta(\mathbf{k}) \operatorname{step}\left(\frac{\mathbf{k}^{\mathrm{r}} \cdot \widehat{\mathbf{o}}}{k_{0}}-\sqrt{1-N_{O}^{2}}\right) .
$$

The contribution of this term to the output is, $\tilde{O}_{S S}(\mathbf{k})=$ $\int \tilde{I}_{S S}(\mathbf{k})\left|\tilde{G}_{I}\left(-\mathbf{k}_{\mathbf{r}}\right)\right| \mathrm{d}^{3} k_{r}$, such that,

$$
\begin{gathered}
\tilde{O}_{S S}(\mathbf{k})=\delta(\mathbf{k}) \frac{\left|R E_{r}\right|^{2}}{4 \pi k_{0}} \int \operatorname{step}\left(\frac{\mathbf{k}^{\mathrm{r}} \cdot \widehat{\mathbf{o}}}{k_{0}}-\sqrt{1-N_{O}^{2}}\right) \times \\
\operatorname{step}\left(\frac{-\mathbf{k}_{\mathbf{r}} . \widehat{\mathbf{o}}}{k_{0}}-\sqrt{1-N_{I}^{2}}\right) \delta\left(\left|\mathbf{k}_{\mathbf{r}}\right|-k_{0}\right) \mathrm{d}^{3} k_{r} .
\end{gathered}
$$

\section{The $\tilde{O}_{S D}(\mathbf{k})$ term:}

The integral $\tilde{I}_{S D}(\mathbf{k})=\int \tilde{E}_{S}\left(\mathbf{k}^{\prime}\right) \tilde{E}_{D}^{*}\left(\mathbf{k}^{\prime}-\mathbf{k}\right) \mathrm{d}^{3} k^{\prime}$, is given by,

$$
\begin{gathered}
\tilde{I}_{S D}(\mathbf{k})=\int R E_{r} \operatorname{step}\left(\frac{\mathbf{k}^{\prime} . \widehat{\mathbf{o}}}{k_{0}}-\sqrt{1-N_{O}^{2}}\right) \delta\left(\mathbf{k}^{\prime}-\mathbf{k}^{\mathrm{r}}\right) \times \\
E_{r}^{*} \pi j \tilde{G}_{0}^{*}\left(\mathbf{k}^{\prime}-\mathbf{k}\right)\left|\mathbf{k}^{\prime}-\mathbf{k}-\mathbf{k}_{\mathbf{r}}\right|^{2} \widetilde{\Delta}_{S}^{*}\left(\mathbf{k}^{\prime}-\mathbf{k}-\mathbf{k}_{\mathbf{r}}\right) \mathrm{d}^{3} k^{\prime} .
\end{gathered}
$$

Using $\tilde{G}^{*}{ }_{O}(\mathbf{k})=-\tilde{G}_{O}(\mathbf{k})$ and $\widetilde{\Delta}^{*}(\mathbf{k})=-\frac{R^{*}}{R} \widetilde{\Delta}_{S}(-\mathbf{k}) \quad$ direct integration gives,

$$
\tilde{I}_{S D}(\mathbf{k})=\left|E_{r}\right|^{2} \pi j R^{*} \tilde{G}_{O}\left(\mathbf{k}^{\mathrm{r}}-\mathbf{k}\right)\left|\mathbf{k}^{\mathrm{r}}-\mathbf{k}-\mathbf{k}_{\mathbf{r}}\right|^{2} \times
$$




$$
\operatorname{step}\left(\frac{\mathbf{k}^{\mathrm{r} . \widehat{o}}}{k_{0}}-\sqrt{1-N_{O}^{2}}\right) \widetilde{\Delta}_{S}\left(\mathbf{k}^{\mathbf{r}}+\mathbf{k}+\mathbf{k}_{\mathbf{r}}\right) .
$$

The contribution of this term to the output is, $\tilde{O}_{S D}(\mathbf{k})=$ $\int \tilde{I}_{S D}(\mathbf{k})\left|\tilde{G}_{I}\left(-\mathbf{k}_{\mathbf{r}}\right)\right| \mathrm{d}^{3} k_{r}$, such that,

$$
\begin{gathered}
\tilde{O}_{S D}(\mathbf{k})=\frac{-\left|E_{r}\right|^{2} \pi R^{*}}{\left(4 \pi k_{0}\right)^{2}} \int \delta\left(\left|\mathbf{k}^{\mathbf{r}}-\mathbf{k}\right|-k_{0}\right) \operatorname{step}\left(\frac{\left(\mathbf{k}^{\mathrm{r}}-\mathbf{k}\right) \cdot \widehat{\mathbf{o}}}{k_{0}}-\sqrt{1-N_{O}^{2}}\right) \\
\times\left|\mathbf{k}+\left(\mathbf{2} \mathbf{k}_{\mathbf{r}} \cdot \mathbf{z}\right) \mathbf{z}\right|^{2} \operatorname{step}\left(\frac{\mathbf{k}^{\mathrm{r}} \cdot \widehat{\mathbf{o}}}{k_{0}}-\sqrt{1-N_{O}^{2}}\right) \widetilde{\Delta}_{S}\left(\mathbf{k}+\left(\mathbf{2} \mathbf{k}_{\mathbf{r}} \cdot \mathbf{z}\right) \mathbf{z}\right) \\
\quad \times \delta\left(\left|\mathbf{k}_{\mathbf{r}}\right|-k_{0}\right) \operatorname{step}\left(\frac{-\mathbf{k}_{\mathbf{r}} \cdot \widehat{\mathbf{o}}}{k_{0}}-\sqrt{1-N_{I}^{2}}\right) \mathrm{d}^{3} k_{r} .
\end{gathered}
$$

Writing the integral in terms of the reflected component $\mathbf{k}_{\mathbf{r}}=\mathbf{k}^{\mathrm{r}}-2\left(\mathbf{k}^{\mathrm{r}} \cdot \mathbf{z}\right) \mathbf{z}$ and noting that $\mathrm{d}^{3} k_{r}=-\mathrm{d}^{3} k^{r}$, we have,

$$
\begin{aligned}
& \tilde{O}_{S D}(\mathbf{k})=\frac{\left|E_{r}\right|^{2} \pi R^{*}}{\left(4 \pi k_{0}\right)^{2}} \int \delta\left(\left|\mathbf{k}^{\mathrm{r}}-\mathbf{k}\right|-k_{0}\right) \operatorname{step}\left(\frac{\left(\mathbf{k}^{\mathrm{r}}-\mathbf{k}\right) \cdot \widehat{\mathbf{o}}}{k_{0}}-\sqrt{1-N_{O}^{2}}\right) \\
& \quad \times\left|\mathbf{k}-\left(2 \mathbf{k}^{\mathrm{r}} \cdot \mathbf{z}\right) \mathbf{z}\right|^{2} \operatorname{step}\left(\frac{\mathbf{k}^{\mathrm{r}} \cdot \widehat{\mathbf{o}}}{k_{0}}-\sqrt{1-N_{O}^{2}}\right) \widetilde{\Delta}_{S}\left(\mathbf{k}-\left(2 \mathbf{k}^{\mathrm{r}} \cdot \mathbf{z}\right) \mathbf{z}\right) \\
& \quad \times \delta\left(\left|\mathbf{k}^{\mathrm{r}}\right|-k_{0}\right) \operatorname{step}\left(\frac{-\left(\mathbf{k}^{\mathrm{r}}-\left(2 \mathbf{k}^{\mathrm{r}} \cdot \mathbf{z}\right) \mathbf{z}\right) \cdot \widehat{\mathbf{o}}}{k_{0}}-\sqrt{1-N_{I}^{2}}\right) \mathrm{d}^{3} k^{r} . \quad(\mathrm{B} 16)
\end{aligned}
$$

Using the substitution $\delta\left(\left|\mathbf{k}^{\mathrm{r}}\right|-k_{0}\right) \delta\left(\left|\mathbf{k}^{\mathrm{r}}-\mathbf{k}\right|-k_{0}\right)=$ $\frac{k_{0}^{2}}{\left|k_{0 z}^{r} k_{x}-k_{0 x}^{r} k_{z}\right|} \delta\left(k_{x}^{\mathrm{r}}-k_{0 x}^{r}\right) \delta\left(k_{z}^{\mathrm{r}}-k_{0 z}^{r}\right)$ (see APPENDIX A, Eq. (A9), and noting that $\widetilde{\Delta}_{S}(\mathbf{k})$ has no dependence on $k_{z}$ such that $\widetilde{\Delta}_{S}\left(\mathbf{k}-\left(\mathbf{2} \mathbf{k}^{\prime} \cdot \mathbf{z}\right) \hat{\mathbf{z}}\right)=\widetilde{\Delta}_{S}(\mathbf{k})$, we can write,

$$
\begin{gathered}
\tilde{O}_{S D}(\mathbf{k})=\widetilde{\Delta}_{S}(\mathbf{k}) \frac{\left|E_{r}\right|^{2} R^{*} \pi}{(4 \pi)^{2}} \int \mathrm{d}^{3} k^{r} \frac{1}{\left|k_{0 z}^{r} k_{x}-k_{0 x}^{r} k_{z}\right|} \times \\
\delta\left(k_{x}^{\mathrm{r}}-k_{0 x}^{r}\right) \delta\left(k_{z}^{\mathrm{r}}-k_{0 z}^{r}\right) \operatorname{step}\left(\frac{\left(\mathbf{k}^{\mathrm{r}}-\mathbf{k}\right) \cdot \widehat{\mathbf{o}}}{k_{0}}-\sqrt{1-N_{O}^{2}}\right) \times \\
\left|\mathbf{k}-\left(\mathbf{2} \mathbf{k}^{\mathrm{r}} \cdot \mathbf{z}\right) \mathbf{z}\right|^{2} \operatorname{step}\left(\frac{\mathbf{k}^{\mathrm{r}} \cdot \widehat{\mathbf{o}}}{k_{0}}-\sqrt{1-N_{O}^{2}}\right) \times \\
\operatorname{step}\left(\frac{-\left(\mathbf{k}^{\mathrm{r}}-\left(2 \mathbf{k}^{\mathrm{r}} \cdot \mathbf{z}\right) \mathbf{z}\right) \cdot \widehat{\mathbf{o}}}{k_{0}}-\sqrt{1-N_{I}^{2}}\right) .
\end{gathered}
$$

Finally after integrating over $\mathrm{d} k_{x}^{\mathrm{r}} \mathrm{d} k_{z}^{\mathrm{r}}$ we will have $\mathbf{k}_{0}^{\mathrm{r}}=$ $\left(k_{0 x}^{r}, k_{y}^{r}, k_{0 z}^{r}\right)$ and we can write,

$$
\tilde{O}_{S D}(\mathbf{k})=\widetilde{\Delta}_{S}(\mathbf{k}) H_{S D}(\mathbf{k})
$$

$$
\begin{aligned}
& \widetilde{H}_{S D}(\mathbf{k})=\frac{\left|E_{r}\right|^{2} R^{*}}{16 \pi} \int \mathrm{d} k_{y}^{r} \frac{\left|\mathbf{k}-\left(2 \mathbf{k}_{0}^{\mathrm{r}} \cdot \mathbf{z}\right) \mathbf{z}\right|^{2}}{|\mathbf{k}| \sqrt{k_{0}^{2}-|\mathbf{k}|^{2} / 4-k_{y}^{\mathrm{r}}}} \times \\
& \operatorname{step}\left(\frac{k_{0 x}^{r} o_{x}+k_{0 z}^{r} o_{z}-k_{x} o_{x}-k_{z} o_{z}}{k_{0}}-\sqrt{1-N_{O}^{2}}\right) \times \\
& \operatorname{step}\left(\frac{k_{0 \chi}^{r} o_{\chi}+k_{0 Z}^{r} o_{Z}}{k_{0}}-\sqrt{1-N_{O}^{2}}\right) \operatorname{step}\left(\frac{-k_{0 x}^{r} o_{\chi}+k_{0 Z}^{r} o_{Z}}{k_{0}}-\sqrt{1-N_{I}^{2}}\right) \text {. }
\end{aligned}
$$

And it is noted that,

$$
\begin{aligned}
& k_{0 x}^{r}=\frac{1}{2}\left(k_{x}-\frac{k_{x} k_{z}}{\left|k_{x}\right||\mathbf{k}|} \sqrt{4 k_{N}^{2}-|\mathbf{k}|^{2}}\right), \\
& k_{0 z}^{r}=\frac{1}{2}\left(k_{z}+\frac{\left|k_{x}\right|}{|\mathbf{k}|} \sqrt{4 k_{N}^{2}-|\mathbf{k}|^{2}}\right)
\end{aligned}
$$

where $k_{N}^{2}=k_{0}^{2}-k_{y}^{r^{2}}$.

\section{The $\tilde{O}_{D S}(\mathbf{k})$ term:}

A similar analysis provides the $\widetilde{O}_{D S}(\mathbf{k})$ term such that,

$$
\begin{gathered}
\tilde{O}_{D S}(\mathbf{k})=\widetilde{\Delta}_{S}(\mathbf{k}) H_{D S}(\mathbf{k}) \text {, where, } \\
\widetilde{H}_{S D}(\mathbf{k})=-\frac{\left|E_{r}\right|^{2} R^{*}}{16 \pi} \int \mathrm{d} k_{y}^{m} \frac{\left|\mathbf{k}+\left(\mathbf{2} \mathbf{k}_{0}^{m} \cdot \mathbf{z}\right) \mathbf{z}\right|^{2}}{|\mathbf{k}| \sqrt{k_{0}^{2}-|\mathbf{k}|^{2} / 4-k_{y}^{m^{2}}}} \times \\
\operatorname{step}\left(\frac{k_{0 x}^{m} o_{x}+k_{0 z}^{m} o_{z}+k_{x} o_{x}+k_{z} o_{z}}{k_{0}}-\sqrt{1-N_{O}^{2}}\right) \times \\
\operatorname{step}\left(\frac{k_{0 x}^{m} o_{x}+k_{0 Z}^{m} o_{Z}}{k_{0}}-\sqrt{1-N_{O}^{2}}\right) \operatorname{step}\left(\frac{-k_{0 x}^{m} o_{x}+k_{0 Z}^{m} o_{z}}{k_{0}}-\sqrt{1-N_{I}^{2}}\right),
\end{gathered}
$$

where components of the vector $\mathbf{k}_{0}^{\mathrm{m}}=\left(k_{0 x}^{m}, k_{y}^{m}, k_{0 z}^{m}\right)$ are:

$$
\begin{aligned}
& k_{0 x}^{m}=\frac{1}{2}\left(-k_{x}-\frac{k_{x} k_{z}}{\left|k_{x}\right||\mathbf{k}|} \sqrt{4 k_{N}^{2}-|\mathbf{k}|^{2}}\right), \\
& k_{0 z}^{m}=\frac{1}{2}\left(-k_{z}+\frac{\left|k_{x}\right|}{|\mathbf{k}|} \sqrt{4 k_{N}^{2}-|\mathbf{k}|^{2}}\right),
\end{aligned}
$$

where $k_{N}^{2}=k_{0}^{2}-k_{y}^{m^{2}}$.

\section{The $\widetilde{O}_{D D}(\mathbf{k})$ term:}

The integral, $\tilde{I}_{D D}(\mathbf{k})=\int \tilde{E}_{D}\left(\mathbf{k}^{\prime}\right) \tilde{E}_{D}^{*}\left(\mathbf{k}^{\prime}-\mathbf{k}\right) \mathrm{d}^{3} k^{\prime}$, is given by,

$$
\begin{gathered}
\tilde{I}_{D D}(\mathbf{k})=\frac{\left|E_{r}\right|^{2}}{16 k_{0}^{2}} \int \mathrm{d}^{3} k^{\prime}\left|\mathbf{k}^{\prime}-\mathbf{k}_{\mathbf{r}}\right|^{2}\left|\mathbf{k}^{\prime}-\mathbf{k}-\mathbf{k}_{\mathbf{r}}\right|^{2} \times \\
\widetilde{\Delta}_{S}\left(\mathbf{k}^{\prime}-\mathbf{k}_{\mathbf{r}}\right) \widetilde{\Delta}_{S}^{*}\left(\mathbf{k}^{\prime}-\mathbf{k}-\mathbf{k}_{\mathbf{r}}\right) \delta\left(\left|\mathbf{k}^{\prime}\right|-k_{0}\right) \delta\left(\left|\mathbf{k}^{\prime}-\mathbf{k}\right|-k_{0}\right) \times \\
\operatorname{step}\left(\frac{\mathbf{k}^{\prime} . \widehat{\mathbf{o}}}{k_{0}}-\sqrt{1-N_{O}^{2}}\right) \operatorname{step}\left(\frac{\left(\mathbf{k}^{\prime}-\mathbf{k}\right) . \widehat{\mathbf{o}}}{k_{0}}-\sqrt{1-N_{O}^{2}}\right) .
\end{gathered}
$$

Now we will use again:

$$
\delta\left(\left|\mathbf{k}^{\prime}\right|-k_{0}\right) \delta\left(\left|\mathbf{k}^{\prime}-\mathbf{k}\right|-k_{0}\right)=
$$

where, 


$$
\begin{gathered}
=\frac{k_{0}^{2}}{|\mathbf{k}| \sqrt{k_{0}^{2}-|\mathbf{k}|^{2} / 4-k_{y}^{\prime 2}}} \delta\left(k_{x}^{\prime}-k_{0 x}^{\prime}\right) \delta\left(k_{z}^{\prime}-k_{0 z}^{\prime}\right) \\
\tilde{I}_{D D}(\mathbf{k})=\frac{\left|E_{r}\right|^{2}}{16 k_{0}^{2}} \int \mathrm{d}^{3} k^{\prime} \frac{k_{0}^{2} \delta\left(k_{x}^{\prime}-k_{0 x}^{\prime}\right) \delta\left(k_{z}^{\prime}-k_{0 z}^{\prime}\right)}{|\mathbf{k}| \sqrt{k_{0}^{2}-\frac{|\mathbf{k}|^{2}}{4}-k_{y}^{\prime 2}} \times} \\
\left|\mathbf{k}^{\prime}-\mathbf{k}_{\mathbf{r}}\right|^{2}\left|\mathbf{k}^{\prime}-\mathbf{k}-\mathbf{k}_{\mathbf{r}}\right|^{2} \widetilde{\Delta}_{S}\left(\mathbf{k}^{\prime}-\mathbf{k}_{\mathbf{r}}\right) \widetilde{\Delta}_{S}^{*}\left(\mathbf{k}^{\prime}-\mathbf{k}-\mathbf{k}_{\mathbf{r}}\right) \times \\
\operatorname{step}\left(\frac{\mathbf{k}^{\prime} . \widehat{\mathbf{o}}}{k_{0}}-\sqrt{1-N_{O}^{2}}\right) \operatorname{step}\left(\frac{\left(\mathbf{k}^{\prime}-\mathbf{k}\right) . \widehat{o}}{k_{0}}-\sqrt{1-N_{O}^{2}}\right)
\end{gathered}
$$

After integrating along variables $k_{x}^{\prime}$ and $k_{z}^{\prime}$ we have,

$$
\begin{gathered}
\tilde{I}_{D D}(\mathbf{k})=\frac{\left|E_{r}\right|^{2}}{16|\mathbf{k}|} \int \frac{\mathrm{d} k_{y}^{\prime}}{\sqrt{k_{0}^{2}-\frac{|\mathbf{k}|^{2}}{4}-k_{y}^{\prime 2}}} \times \\
\left|\mathbf{k}_{\mathbf{0}}^{\prime}-\mathbf{k}_{\mathbf{r}}\right|^{2}\left|\mathbf{k}_{\mathbf{0}}^{\prime}-\mathbf{k}-\mathbf{k}_{\mathbf{r}}\right|^{2} \widetilde{\Delta}_{S}\left(\mathbf{k}_{\mathbf{0}}^{\prime}-\mathbf{k}_{\mathbf{r}}\right) \widetilde{\Delta}_{S}^{*}\left(\mathbf{k}_{\mathbf{0}}^{\prime}-\mathbf{k}-\mathbf{k}_{\mathbf{r}}\right) \times \\
\operatorname{step}\left(\frac{\mathbf{k}_{\mathbf{0}}^{\prime} \mathbf{0}}{k_{0}}-\sqrt{1-N_{O}^{2}}\right) \operatorname{step}\left(\frac{\left(\mathbf{k}_{\mathbf{0}}^{\prime}-\mathbf{k}\right) . \hat{\mathbf{o}}}{k_{0}}-\sqrt{1-N_{O}^{2}}\right)
\end{gathered}
$$

where $\mathbf{k}_{0}^{\prime}=\left(k_{0 x}^{\prime}, k_{y}^{\prime}, k_{0 z}^{\prime}\right)$ (as defined in (A7), (A8), (A12)). The contribution of this term to the output is, $\tilde{O}_{D D}(\mathbf{k})=$ $\int \tilde{I}_{D D}(\mathbf{k})\left|\tilde{G}_{I}\left(-\mathbf{k}_{\mathbf{r}}\right)\right| \mathrm{d}^{3} k_{r}$, such that,

$$
\begin{gathered}
\tilde{O}_{D D}(\mathbf{k})=\frac{1}{16 \pi^{2}|\mathbf{k}|} \int \frac{\mathrm{d} k_{y}^{\prime}}{\sqrt{k_{0}^{2}-\frac{|\mathbf{k}|^{2}}{4}-k_{y}^{\prime 2}}} \times \\
\operatorname{step}\left(\frac{\mathbf{k}_{\mathbf{0}}^{\prime} \cdot \widehat{\mathbf{o}}}{k_{0}}-\sqrt{1-N_{O}^{2}}\right) \operatorname{step}\left(\frac{\left(\mathbf{k}_{\mathbf{0}}^{\prime}-\mathbf{k}\right) \cdot \widehat{\mathbf{o}}}{k_{0}}-\sqrt{1-N_{O}^{2}}\right) \times \\
\frac{\left|E_{r}\right|^{2} \pi}{4 k_{0}} \int \mathrm{d}^{3} k_{r}\left|\mathbf{k}_{\mathbf{0}}^{\prime}-\mathbf{k}_{\mathbf{r}}\right|^{2}\left|\mathbf{k}_{\mathbf{0}}^{\prime}-\mathbf{k}-\mathbf{k}_{\mathbf{r}}\right|^{2} \widetilde{\Delta}_{S}\left(\mathbf{k}_{\mathbf{0}}^{\prime}-\mathbf{k}_{\mathbf{r}}\right) \times \\
\widetilde{\Delta}_{S}^{*}\left(\mathbf{k}_{\mathbf{0}}^{\prime}-\mathbf{k}-\mathbf{k}_{\mathbf{r}}\right) \operatorname{step}\left(\frac{-\mathbf{k}_{\mathbf{r}} \widehat{\mathbf{o}}}{k_{0}}-\sqrt{1-N_{I}^{2}}\right) \delta\left(\left|\mathbf{k}_{\mathbf{r}}\right|-k_{0}\right), \quad(\mathrm{B} 2
\end{gathered}
$$

where the components of the vector $\mathbf{k}_{0}^{\prime}=\left(k_{0 x}^{\prime}, k_{y}^{\prime}, k_{0 z}^{\prime}\right)$ are,

$$
\begin{aligned}
& k_{0 x}^{\prime}=\frac{1}{2}\left(k_{x}-\frac{k_{x} k_{z}}{\left|k_{x}\right||\mathbf{k}|} \sqrt{4 k_{N}^{2}-|\mathbf{k}|^{2}}\right), \\
& k_{0 z}^{\prime}=\frac{1}{2}\left(k_{z}+\frac{\left|k_{x}\right|}{|\mathbf{k}|} \sqrt{4 k_{N}^{2}-|\mathbf{k}|^{2}}\right), \\
& \text { where } k_{N}^{2}=k_{0}^{2}-k_{y}^{\prime 2} .
\end{aligned}
$$

